\title{
Multichannel Staggered SAR Azimuth Processing
}

\author{
Felipe Queiroz de Almeida, Marwan Younis, Senior Member, IEEE, \\ Gerhard Krieger, Fellow, IEEE and Alberto Moreira, Fellow, IEEE
}

\begin{abstract}
State-of-the-art and future spaceborne SAR systems increasingly often face the requirement of providing highresolution images with reduced revisit times, requiring coverage of wide swaths. Since these are contradicting drivers in terms of system design, different alternatives for High-Resolution Wide Swath (HRWS) SAR imaging have been investigated, relying on digital beamforming (DBF) and the use of multiple receiver channels, both in elevation and azimuth dimensions. In this context, Staggered SAR, which operates with a pulse repetition frequency variation, using a single channel in azimuth proves itself as a promising alternative for covering wide continuous swaths with moderate azimuth resolution, whereas the use of multiple azimuth receiver channels bears the potential of improving the azimuth resolution over a given swath, but has yet only been applied to systems with a fixed pulse repetition frequency. This paper introduces and analyzes in detail processing techniques suitable for the combination of these techniques, leading to novel multichannel Staggered SAR imaging modes with the potential for very fine azimuth resolution over ultra-wide swaths. A system concept with $2.0 \mathrm{~m}$ azimuth resolution over a $400 \mathrm{~km}$ swath in quad-pol is provided as an example.
\end{abstract}

Index Terms - Radar, Radar imaging, Spaceborne radar, Synthetic aperture radar, Digital beamforming, High resolution wide swath

\section{INTRODUCTION}

$\mathrm{R}$ EMOTE sensing of the Earth by means of spaceborne Synthetic Aperture Radar (SAR) has received great interest in recent years, owing to the numerous possible applications and virtually weather- and daylight independent operation. Clearly, both high (spatial) image resolution and short revisit times (equivalent to a high temporal resolution) translate into a larger information content which is highly desirable for numerous applications. State-of-the-art and next-generation SAR systems [1],[2],[3] are in fact required to simultaneously provide high spatial and temporal resolutions in order to enable nearlycontinuous scientific observation of important dynamic Earth processes.

Manuscript received December 14, 2016. F. Queiroz de Almeida, M. Younis, G. Krieger and A. Moreira are with the Microwaves and Radar Institute of the German Aerospace Center (DLR), Oberpfaffenhofen, Germany (e-mail: felipe.queirozdealmeida@dlr.de.).
The requirement of a short revisit time can only be fulfilled if an adequately wide swath is imaged. This needs to be done without sacrificing the high spatial resolution, which poses a challenging problem to system design, as a compromise between swath width and azimuth resolution is inherent to conventional single-channel SAR systems [4],[5],[6]. High-Resolution WideSwath (HRWS) SAR imaging [4],[5],[7],[8],[9],[10],[11],[12], has thus become a very active research topic, in order to enable system design to achieve the demanding goals of near-future SAR missions.

Digital beamforming and the use of multiple channels in both elevation and azimuth dimensions play an important role in the extension of the system capabilities. In particular, systems with multiple receive channels in azimuth allow a gain in the sampling rate over azimuth, thus supporting an improved azimuth resolution compared to single-channel SAR systems, without an increase of the pulse repetition frequency (PRF). This is illustrated conceptually in Fig.1 (a) and in practice, i.e. for realistic antenna lengths, enables the acquisition of moderately wide swaths with high azimuth resolution [9],[10]. From [10]:

$P R F<\frac{c_{0}}{2 \cdot W_{\text {swath }} \cdot \sin \left(\eta_{\text {inc }}\right)}$,

where $c_{0}$ is the speed of light, $\eta_{i n c}$ is the incidence angle and $W_{\text {swath }}$ is the swath width on ground. Moreover, assuming the uniform sampling condition as reference, the azimuth antenna length should be in the order of

$L_{\text {ant }}=\frac{2 \cdot v_{\text {sat }}}{P R F}$,

where $v_{\text {sat }}$ is the satellite velocity. This in turn means that

$W_{\text {swath }}<\frac{c_{0} \cdot L_{\text {ant }}}{4 \cdot v_{\text {sat }} \cdot \sin \left(\eta_{\text {inc }}\right)}$,

and shows more clearly the reason for the limitation in the swath width, namely that the azimuth antenna length tends to become too large for space deployment in the case of very low PRF, as required by ultra-wide swaths. For instance, to keep the antenna length smaller than $20 \mathrm{~m}$, assuming $v_{\text {sat }}=7 \mathrm{~km} / \mathrm{s}$ and $\eta_{\text {inc }}=60^{\circ}$, the maximum swath width is limited to $236 \mathrm{~km}$. Conversely, a $32.4 \mathrm{~m}$ long antenna operated at $P R F=433 \mathrm{~Hz}$ would be required to achieve a swath of $400 \mathrm{~km}$. 
For a fixed PRF given by the maximum achievable antenna length, extending the imaged swath eventually leads to echo signals from different portions of the swath being received simultaneously. This phenomenon is known as range ambiguities in conventional SAR. In this context, the usage of SCORE (SCan-On-Receive [13]) with multiple simultaneous elevation beams [9],[10] is an interesting alternative, allowing the swath to be extended beyond the limits imposed by range ambiguities. The basic principle is namely that, even though simultaneous, the main signal and the ambiguities show different directions of arrival. They may thus be separated relying on digital elevation beamforming, provided that the system architecture allows several beams to be formed simultaneously by means of different weightings. This can be interpreted as an extension of SCORE to follow the returns on ground in different sub-swaths simultaneously, by using multiple (as many as required by the number of sub-swaths) time-dependent weights, as depicted in Fig.1 (b) for the case of a reflector SAR system. Note that this also implies a new interpretation of range ambiguities, as they become useful signals which are imaged through different subswaths and no longer a nuisance effect.

Though quite effective at increasing the total imaged swath by stacking several sub-swaths, the later technique is subject to a limitation in the sense that the sub-swaths are separated by gaps caused by the transmission events. This cannot be avoided in a fixed-PRF monostatic system, as the echoes cannot be recorded simultaneously with transmission. The gap lengths are given by

$\Delta R_{\text {blind }}=2 \cdot \frac{c \cdot T_{p}}{2}=\frac{c \cdot T_{d c}}{P R F}$,

where $T_{p}$ denotes the pulse length and $T_{d c}$ describes the duty cycle of the system. The factor two accounts for the full reception of the echoes of duration $T_{p}$, hence the duration refers to gaps in the full range-resolution image. If e.g. $T_{d c}=10 \%$ the blind ranges achieve a length of $12 \mathrm{~km}$ for a PRF of $2500 \mathrm{~Hz}$.

This however can be avoided by staggering of the system PRF [14],[15]. In a staggered $S A R$ mode, the pulse repetition interval (PRI) is changed cyclically from pulse to pulse. The blockage of the receive signal by transmission events still occurs, but at different ranges for each azimuth position. This creates a blockage pattern which no longer shows blind ranges at fixed range positions (in the sense of all azimuth pulses being lost at particular ranges) but rather lost pulses which are distributed over the swath in a cyclical azimuth-dependent manner (cf. Fig.1 (c)). In [11],[15],[16] a design procedure for the sequences is reported, which allows after interpolation the recovery of data equivalent to a conventional single azimuth channel SAR acquisition. This approach enables a continuous gapless wide swath, a feature typically highly desirable from the perspective of applications, at the price of increased system complexity. The maximum azimuth resolution of this class of system is nonetheless limited, since a single azimuth channel is employed.

Given the limitations of the aforementioned approach, resorting to the use of multiple azimuth channels in combination with staggered SAR - as illustrated conceptually in Fig.1 (d) presents itself as an opportunity for image acquisitions with very high resolution over ultra-wide swaths, combining the strength of both methods. The signal processing for such a system is however challenging: for instance, whenever the timing dictates the loss of a pulse, a $N_{c h}$ channel system also loses $N_{c h}$ samples, amplifying the non-uniformity. In addition, new techniques are required, as the conventional multichannel processing strategies rely on frequency-domain processing, which cannot be straightforwardly extended to a staggered SAR system due to the PRI variation.

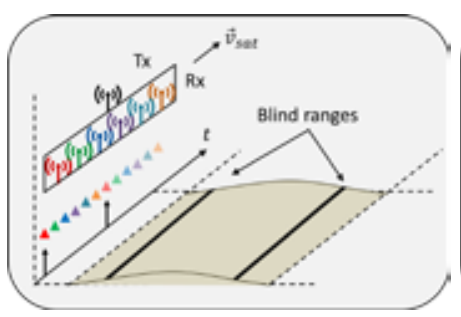

(a)

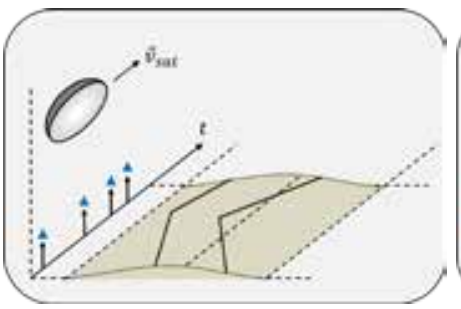

(c)

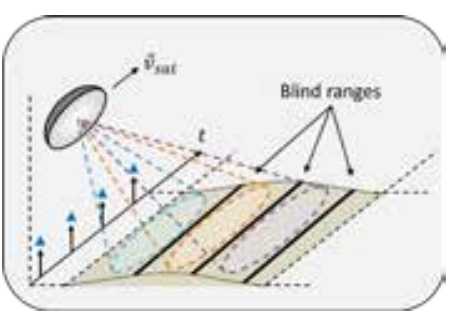

(b)

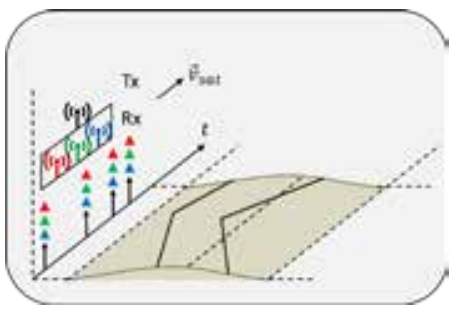

(d)
Fig.1: Schematic representation of HRWS concepts and imaging modes. (a) shows a system with multiple azimuth channels on receive, enabling a finer sampling of the signal and thus supporting a higher resolution; (b) illustrates the formation of multiple elevation beams, combining simultaneously imaged subswaths separated by blind ranges related to transmission events which are azimuth invariant, due to the uniform pulse repetition interval (PRI); (c) shows a staggered SAR system, in which the different PRIs cause the transmission-induced blockage to affect different ranges for each azimuth position, allowing posterior interpolation and recovery; (d) shows a multichannel staggered-PRI system, which is a combination of (a) and (c) in the azimuth dimension.

This paper closely examines new techniques suitable for processing multichannel staggered SAR modes - briefly introduced in [17] and object of [18] - and provides different examples of the capabilities of these techniques. Simulations show the feasibility of imaging a $400 \mathrm{~km}$ wide continuous swath with up to $2.0 \mathrm{~m}$ azimuth resolution.

\section{A. Paper Structure}

The rest of this paper is organized as follows: Section II describes the modelling of the problem from a processing perspective (Section II.A) and the different digital beamforming algorithms developed to solve it (Sections II.B-II.G), starting from the general framework. Sections III and IV.A present simulation results showing the performance of the methods. On the one hand, Section III examines closely a particular case of the resampling operation, giving insight into the processing and 
the performance trade-offs made possible by adjusting the method's parameters. Section IV.B on the other hand focuses on system design and performance analysis over a swath of interest, showing how the methods can be used to fulfil demanding HRWS mission requirements. Finally, a review and summary are provided in Section V.

\section{Multichannel StAggered SAR AND THE ReQuiRED AZIMUTH RESAMPLING}

This section starts by presenting the modeling of the Staggered SAR resampling problem in Section II.A, followed by a general framework for its solution in Section II.B. Section II.C presents a first particular solution which is especially important for the physical interpretation of the procedure and discusses its limitations. Section II.D provides then the core description of the proposed method, relying on a novel modelling that transforms the problem at hand into a beamforming problem that can be solved by cost function minimization. Sections II.E and II.F examine in addition two cost function alternatives which particularize the solution presented in Section II.D, in order to account for different aspects of system performance and introduce compromises in the final solution. Finally, Section II.G provides a further refinement of the solution that seeks to give the outputs a more uniform performance, regardless of the choice of the cost function.

\section{A. Problem Overview and Signal Model}

The received azimuth signal of a staggered SAR is sampled in a periodically non-uniform manner and is subject to rangedependent gaps induced by blockage of the received pulses, corresponding to pulse transmission events [11]. Furthermore, a system with multiple azimuth channels is assumed, capable of recording $N_{c h}$ samples per received (or transmitted) pulse. In order to apply conventional SAR processing to such a signal, the task is to convert the effective $N_{e f f}$ staggered PRI pulses per cycle into an output signal that is regularly sampled at a rate of $P R F_{\text {multi }}=N_{c h} \cdot \overline{P R F}_{\text {eff }}$, where $\overrightarrow{P R F}_{\text {eff }}$ is the effective average sampling rate of the pulses, accounting for blockage at the specific range ${ }^{1}$. The desired resampling operation is represented schematically in Fig.2 (a). Out of the $N_{P R I}$ transmitted pulses within a cycle, $N_{e f f} \leq N_{P R I}$ are effectively received, yielding $N_{c h}$ samples each. The goal is to combine a suitable number of azimuth samples within a window of length $N_{\text {win }}$ to recover a uniformly sampled SAR signal composed of $N=N_{\text {eff }} \cdot N_{c h}$ samples per cycle.

\footnotetext{
${ }^{1}$ Note that $N_{\text {eff }}$ varies with range, according to the specific PRI sequence which is adopted, and that range-dependent interpolation is applied. At each range, $\overline{P R F}_{\text {eff }}=\frac{N_{\text {eff }}}{N_{P R I}} \cdot \overline{P R F}$, where $\overline{P R F}$ is the mean PRF of the sequence. As a constant $P R F_{\text {mult } i} \leq N_{c h} \cdot \overline{P R F}$ across the swath is desirable, one may either resample each range to its particular $P R F_{\text {multi }}(R)$ and introduce an interpolation to $N_{c h} \cdot \overline{P R F}$ (for the simulation results in Section IV.B this was done by zero-padding before azimuth compression) or use the method to resample directly to $N_{c h} \cdot \overline{P R F}$ at every range.
}

\section{B. General Framework for Solution}

As the next sections show, the resampling may be achieved computing each output sample as a linear combination of $N_{\text {win }}$ input samples, where the weights depend on the output sample position $k$ within the cycle. In other words,

$s_{\text {out }}[k]=\boldsymbol{w}[k]^{T} \cdot \boldsymbol{s}_{\text {in }}$,

where $s_{\text {out }}[k], 1 \leq k \leq N_{e f f} \cdot N_{c h}$ is a sample in a cycle of the output grid and $\boldsymbol{w}[k]$ and $\boldsymbol{s}_{\boldsymbol{i n}}$ are $N_{\text {win }}$-element complex column vectors representing respectively a set of resampling weights and the signal samples gathered over the input window.

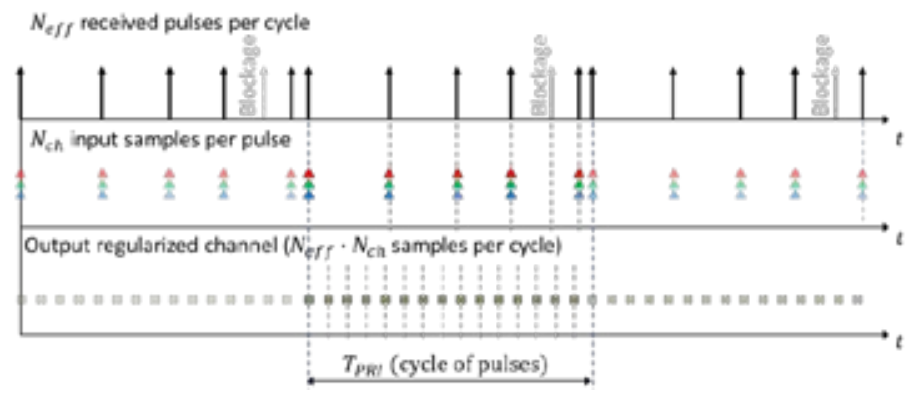

(a)

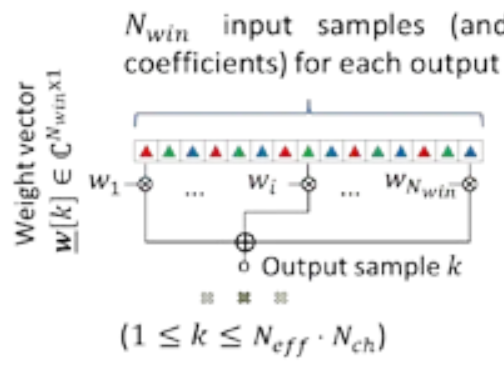

(b)

Fig.2: Required resampling operation focusing on a cycle of pulses of duration $T_{P R I}$. (a) gives an overview showing the input grid composed of a set of $N_{\text {eff }}$ staggered PRI pulses received per cycle, in a multichannel configuration yielding $N_{c h}$ samples per pulse. The overall sampling results in $N_{e f f} \cdot N_{c h}$ samples per cycle, to be rearranged uniformly in the output grid, which is equivalent to a single channel system at a higher sampling rate. (b) focuses on the formation of a single sample of index $k$ in the output grid, which is derived from the combination of $N_{\text {win }}$ input samples with complex weights.

Due to the periodical nature of the sampling of the inputs, a consequence of the cyclical PRI variation, the same set of weights may be applied over several cycles and it is thus sufficient to analyze the outputs over a single period. As elaborated in the next sections, the corresponding weight vector $\underline{\boldsymbol{w}}[k]$ is designed to achieve a suitable phase relation of the output patterns that characterize each sample in a cycle of the output grid. The approach for determining the weights is considered novel, since it uses a physical interpretation of the antenna illumination phase center to obtain a set of desired virtual antenna patterns that correspond to regular sampling and can be synthesized using a modification of known beamforming techniques. Furthermore, the general framework is seen to introduce the possibility of employing information not only from the different channels in a given pulse, as is done in standard 
beamforming techniques $\left(N_{\text {win }}=N_{c h}\right)$, but also take the information from neighboring pulses into consideration. This can be interpreted as the use of virtual antenna patterns (cf. Section II.D) while mathematically being equivalent to a form of interpolation. Note that in the case $N_{\text {win }}<N_{c h} \cdot N_{\text {eff }}$ a sliding neighborhood consisting of an output-position dependent subset of the cycle of pulses is employed as input for the uniformly sampled signal recovery. In the case $N_{\text {win }} \geq N_{c h} \cdot N_{\text {eff }}$ either the full cycle of pulses or more - introducing an overlap between cycles - are employed as inputs.

\section{Resampling through Primary Beam Steering}

Acquisition of the azimuth signal through multiple phase centers [5] is the key to the uniform signal reconstruction capability. In the time domain, the resampling task can be interpreted as positioning a suitable set of phase centers, namely the one that implements the desired regular sampling. In this context, beamforming techniques to alter the available phase center are of interest as possible solutions to the resampling problem under study.

For a reflector antenna uniform array steering [19],[20] with the feed (primary) beam may be employed to obtain patterns with different phase centers which still observe the same Doppler spectrum on the ground. The concept is illustrated schematically in Fig. 3. The phase center diversity is achieved by illuminating different regions of the reflector's surface, by means of which the spatial center of the induced current distributions is altered and so is the secondary pattern's phase center. Illuminating a subset instead of the whole of the reflector narrows the induced current distribution on the reflector's surface and thus broadens the resulting patterns on ground, reducing the gain. Nonetheless, the ability to continuously vary the primary beam's position allows adjustment of the phase centers, which has several potential applications [20].

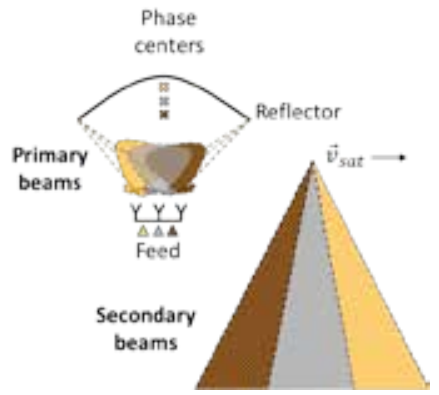

(a)

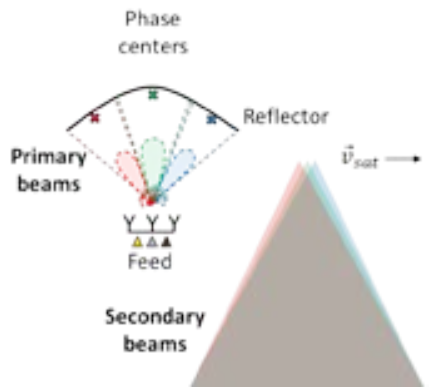

(b)
Fig. 3: Schematic description of phase center diversity by steering the primary beam of a reflector antenna system with a multichannel feed in azimuth. (a) shows in different colors the primary and secondary beams of the individual elements, which illuminate different Doppler regions with the same phase center. (b) shows in different colors the steering of the primary beams to different regions, with the result of broadening the secondary patterns and changing the phase centers.

In particular, this technique can be used to achieve the needed sample regularization and corresponds to a particular case of the aforementioned problem with $N_{\text {win }}=N_{c h}$, meaning conventional beamforming is employed, combining the channels independently for each of the $N_{\text {eff }}$ available pulses. Let the complex patterns of the $N_{c h}$ azimuth channels be denoted by $G_{n}(\theta), 1 \leq n \leq N_{c h}$. The antenna system is thus described by an array manifold vector [21]

$\underline{\boldsymbol{v}}(\theta)=\left[\begin{array}{lll}G_{1}(\theta) & \ldots & G_{N_{c h}}(\theta)\end{array}\right]^{T}$,

which collects the $N_{c h}$ complex antenna patterns as a function of the azimuth angle $\theta$. From classical antenna theory [19],[21], if the feed array is uniform with inter-element spacing $d_{a z}$ in azimuth and $\lambda$ is the wavelength, in order to shift the maximum of the resulting primary pattern $G_{\text {array }}(\theta)=\underline{w}^{\boldsymbol{H}} \cdot \underline{\boldsymbol{v}}(\theta)$ to a certain $\theta_{G O A L}$, a phase-only weighting of the form

$\underline{\boldsymbol{w}}\left(\theta_{G O A L}\right)$

$=\left[\begin{array}{llll}1 & e^{j \cdot \beta\left(\theta_{G O A L}\right)} & \ldots & e^{j \cdot\left(N_{c h}-1\right) \cdot \beta\left(\theta_{G O A L}\right)}\end{array}\right]^{H}$

may be applied, where

$\beta\left(\theta_{G O A L}\right)=\frac{2 \cdot \pi \cdot d_{a z}}{\lambda} \cdot \sin \left(\theta_{G O A L}\right)$,

valid for $\left|\theta_{G O A L}\right| \leq \arcsin \left(\frac{\lambda}{2 \cdot d_{e l}}\right)$.

In order to use this technique, we denote the time instants of the received pulses, which may be obtained by timing analysis of the PRI sequence [11],[15],[16], by $t_{R X}[i], 1 \leq i \leq N_{e f f}$, and the positions of the regular output grid by

$t_{\text {out }}[k]=\frac{k-1}{N_{c h} \cdot \overline{P R F}_{\text {eff }}}$

for $1 \leq k \leq N_{c h} \cdot N_{e f f}$

Assuming a mapping $\theta_{\text {goal }}(\Delta x)$ describing the relationship between the steering angle and the achieved phase center shift is available (this can be obtained by either a geometrical model or fitting of simulations, given knowledge of the complex antenna patterns), one may calculate the set of required shifts

$\Delta x[n, i]=v_{\text {sat }} \cdot\left(t_{\text {out }}\left[N_{c h} \cdot i+n\right]-t_{R X}[i]\right)$

for each $1 \leq i \leq N_{e f f}$ and for $1 \leq n \leq N_{c h}$. Note that this means that the $N_{c h}$ samples from each received pulse - with coinciding phase centers in the reflector case - are used to generate a set of $N_{c h}$ samples which are part of the output grid. The phase center shift required for the samples of each received pulse (index $i$ ) is different, according to the deviation of each pulse's position with respect to the regular grid, as can be observed in Fig.2. By using the mapping $\theta_{\text {goal }}(\Delta x)$ and (7), (8), a set of $N_{e f f} \cdot N_{c h}$ weights of dimension $N_{c h}$ is thus determined, which achieves the resampling.

The phase center diversity relies on changing the position of the illuminated region of the reflector's surface, thus the available span of phase center shifts is inherently limited by the physical dimensions of the reflector. Furthermore, the element patterns of the feed are expected to show some attenuation near the reflector borders, reducing the gain of the steered pattern. 
Border effects contribute further to pattern degradation and performance loss for large phase center shifts, meaning it is desirable to reduce their maximum extent. This can be achieved in the stage of PRI sequence design, by increasing the mean $\mathrm{PRF}$, since in the case of a one-pulse gap, the maximum phase center shift is half of the maximum distance between samples, i.e.

$\Delta x_{\max }<\frac{2 \cdot v_{\text {sat }} \cdot P R I_{\max }}{2}=v_{\text {sat }} \cdot P R I_{\max }$

For instance, for an orbit velocity of $7 \cdot 10^{3} \mathrm{~m} / \mathrm{s}$ and a minimum PRF of $2 \mathrm{kHz}$, a maximum required shift of around $\Delta x= \pm 3.5$ $\mathrm{m}$ is expected, which in turn already requires a rather large reflector of diameter $15 \mathrm{~m}$. Recall that the phase center $p_{c}=$ $\left(p_{c}^{T x}+p_{c}^{R x}\right) / 2$ is not changed on transmit, meaning that only half of the geometrical change translates into a phase center shift. Since the mean PRF cannot be increased without other implications on the system performance, such as e.g. degradation of range ambiguity levels, the azimuth performance of such a system is in last consequence subject to a compromise between the mean PRF and the reflector size, in the sense that a larger reflector supports a wider range of shifts and allows the usage of a lower PRF, though at increased hardware costs.

\section{Resampling through Virtual Beam Synthesis (VBS): General Formulation}

In spite of its possible limitations in the context of this application, the method introduced in Section II.C introduces the interesting concept of using beamforming techniques and exploring the interchangeability between the pattern's phase center and the corresponding sample position to achieve resampling. In this section a method is proposed which builds on these concepts, in the more general scenario of combining $N_{c h}$ channels over an arbitrary number of pulses $N_{p}$, totaling $N_{\text {win }}=N_{p} \cdot N_{c h}$ input samples, as described in Section II.A.

In order to proceed, the different sampling positions over the $N_{p}$ different received pulses must be accounted for. Though only $N_{c h}$ physical channels exist, an extended array manifold vector of length $N_{\text {win }}=N_{p} \cdot N_{c h}$ can be considered for the system, augmenting the manifold vector of the physical channels (6) with a phase ramp describing the pulse position in the sampling over the window. Due to the correspondence between the azimuth angle $\theta$ and the Doppler frequency $f_{d}$, both may be used interchangeably as independent variables of the array manifold. We keep the notation $t_{R X}[i], 1 \leq i \leq N_{p}$ for the time instants of the received pulses, and now express the complex patterns of the $N_{c h}$ azimuth channels by $G_{n}\left(f_{d}\right), 1 \leq n \leq N_{c h}$. The elements of the extended manifold vector $\boldsymbol{v}\left(f_{d}\right)$, which models the input samples over all $N_{e f f} \cdot N_{c h}$ virtual elements, may then be written as

$$
\begin{aligned}
v_{m}\left(f_{d}\right)=G_{k_{1}[m]}\left(f_{d}\right) \cdot & \\
& \exp \left(-j \cdot 2 \cdot \pi \cdot t_{R X}\left[k_{2}[m]\right] \cdot f_{d}\right),
\end{aligned}
$$

for $1 \leq m \leq N_{\text {win }}$, where $k_{1}[m]=1+\left(m \bmod N_{c h}\right)$

$k_{2}[m]=m \% N_{c h}$,

with mod denoting the modulo (integer division remainder) operator, and \% denoting the quotient of integer division. This expresses mathematically that, as $m$ varies, the pattern indices $k_{1}[m]$ vary cyclically from 1 to $N_{c h}$, and the sample indices $k_{2}[m]$ repeat themselves $N_{c h}$ times before being incremented by one. This ensures that all azimuth channels for a given pulse position are taken as part of the manifold. Note that the pulse positions thus translate into an equivalent baseline for the virtual patterns of the extended manifold vector.

It should be recalled that a total of $N_{c h} \cdot N_{\text {eff }}$ unique sample positions per cycle exist, but samples from neighboring cycles may be modelled by considering input sampling instants $t_{R X}[i] \pm n \cdot T_{P R I}, 1 \leq i \leq N_{\text {eff }}$ and integer $n$. Thus, arbitrary input window strategies can be considered by proper implementation of (12).

The desired output samples form as before a regular grid at the increased sampling rate of $N_{\text {out }}=N_{c h} \cdot N_{\text {eff }}$ samples per cycle. This can also be described by a set of $N_{\text {out }}$ output patterns $G_{G O A L}\left(f_{d}, k\right)$, one for each sample $1 \leq k \leq N_{\text {out }}$, with phase relations implied by

$$
\begin{aligned}
G_{G O A L}\left(f_{d}, k\right)= & G_{\text {common }}\left(f_{d}\right) \cdot \\
& \exp \left(-j \cdot 2 \cdot \pi \cdot t_{\text {out }}[k] \cdot f_{d}\right),
\end{aligned}
$$

where $G_{\text {common }}\left(f_{d}\right)$ is the common (i.e. $k$-invariant) component of the patterns of the output samples, representing the desired azimuth pattern of the regular grid which the beamforming is implementing, and

$t_{\text {out }}[k]=\frac{k-1}{N_{c h} \cdot \overline{P R F}_{\text {eff }}}+\delta t$,

denotes the sampling instants of the output grid, being regular by definition. The parameter $\delta t$ is an arbitrary time shift which doesn't change the regularity property of the grid and can be used as a degree of freedom in the design. Minimizing the maximum required sample position shift is a reasonable criterion for its choice. The significance of this parameter is better explained by comparison of two possible choices, as depicted in Fig. 4. The first grid employs $\delta t_{1}=0$, leading to a set of shifts $\Delta t_{1}$ with minimum $\min \left\{\Delta t_{1}\right\}=0$ for the first output sample but a possible large $\max \left\{\Delta t_{1}\right\}$. On the other hand the choice of $\delta t_{2}=0.5 \cdot\left(\min \left\{\Delta t_{1}\right\}+\max \left\{\Delta t_{1}\right\}\right)$ leads to a regular grid 2 whose maximum shift is half of the original one. The azimuth shift introduced by a $\delta t \neq 0$ can be easily compensated at posterior processing steps (for example azimuth compression) after regularization of the data.

One can think of $G_{\text {common }}\left(f_{d}\right)$ in (14) as the desired azimuth pattern of the equivalent single-channel system sampled at $P R F_{\text {multi }}$ which the multichannel system with azimuth beamforming seeks to emulate. The other component, the phase ramps with respect to $t_{\text {out }}[k]$ enforce the regularity of the 
desired output grid, which is necessary for further processing with a conventional SAR processor.

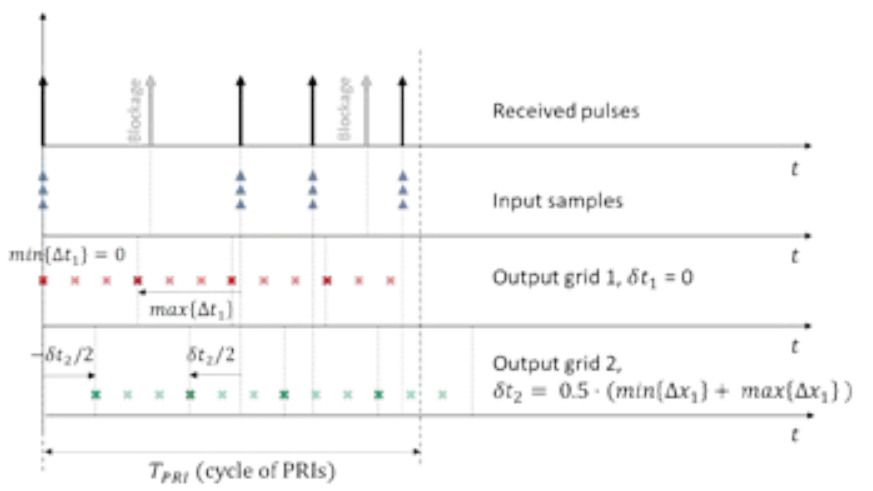

Fig. 4: Comparison of two regular grids (grid 1 and grid 2) highlighting the importance of the parameter $\delta t$ and illustrating the optimum shift minimizing the worst-case phase center shift.

The desired output patterns in (14) represent virtual patterns approximated by the combination of the channels over different pulses (cf. (12)). As $t_{R X}[m]$ changes in the non-uniform input grid, a varying degree of success is obtained (cf. Section II.G) and a residual fluctuation occurs (cf. Fig. 15), but proper design of $G_{\text {common }}\left(f_{d}\right)$ and a suitable level of oversampling on overage allow good resampling performance.

The resampling of the multichannel staggered data is also the goal of the uniform array steering technique in Section II.C, [20], although in that method a single pulse contributes to the beamforming at each time and $N_{p}=1$. In the steering method, the phase relations are determined by the scan angle $\theta_{G O A L}$ to which the feed pattern is steered to; and the resulting illuminated area on the reflector is implicitly defined by the mapping $\theta_{\text {goal }}(\Delta x)$. The patterns achieved by this method also follow the structure of (14) in the sense that they possess a common component which can be assumed to be invariant for small phase center shifts, meaning the pattern is not considerably distorted, in spite of the phase center shift. This means that $G_{\text {common }}\left(f_{d}\right)$ is under certain conditions equal to the sum pattern of all physical channels

$G_{\text {sum }}\left(f_{d}\right)=\frac{1}{N_{c h}} \cdot \sum_{i=1}^{N_{c h}} G_{i}\left(f_{d}\right)$

a pattern which effectively corresponds to steering to the center of the reflector and results in a broader secondary pattern that illuminates approximately the combined beamwidth of the individual elements. The sum pattern of the physical channels remains a reasonable choice for the design of $G_{\text {common }}\left(f_{d}\right)$ in (14), though this degree of freedom may also be exploited (cf. Section II.G). It should be noted that the proposed modeling of each sample by means of an equivalent pattern through (12) and (14) effectively transforms the initial resampling problem of Section II.A into a more tractable pattern synthesis problem. The method is for this reason referred to as the virtual beam synthesis
(VBS) method, and has as main feature the representation of the input and output samples by means of their corresponding patterns, which are considered to be elements of an extended manifold vector. The patterns may be referred to as "virtual" in the sense that they do not represent physical array antenna elements, but rather mathematical constructs incorporating the information of the antenna patterns alongside the timing of the pulses.

What remains to be answered is how to determine the weights that map the inputs modeled by (12) into the outputs modeled by (14). In general, we propose to solve this problem by adaptive beamforming which minimizes a cost function of the form

$\xi\left(G_{G O A L}\left(f_{d}, k\right), v_{i}\left(f_{d}\right)\right)$,

$1 \leq i \leq N_{\text {win }}, 1 \leq k \leq N_{\text {out }}$.

Particular choices of the cost function will give rise to variants of the method, which allow emphasizing certain properties of the solution and introducing compromises if necessary, as will be made clear in the next two sections.

\section{E. Mean Squared Error (MSE) Cost Function}

The first choice of the cost function (17) is based on the leastsquares (LS) pattern synthesis [21] technique. The original LS pattern synthesis problem may be described as follows: given an arbitrary $N$-element array manifold vector $\underline{\boldsymbol{v}}\left(f_{d}\right)$, derive the beamforming weight vector $\boldsymbol{w}$ that leads to the closest approximation $\widehat{G}_{G O A L}\left(f_{d}\right)=\underline{\boldsymbol{w}}^{\overline{\boldsymbol{H}}} \cdot \underline{\boldsymbol{v}}\left(f_{d}\right)$ of a desired pattern $G_{G O A L}\left(f_{d}\right)$. The solution is achieved by minimizing a cost function that measures the integral of the mean squared error (MSE) between the goal pattern and the approximation, i.e.,

$\xi_{M S E}=\int_{f_{1}}^{f_{2}}\left(G_{G O A L}\left(f_{d}\right)-\underline{\boldsymbol{w}}^{\boldsymbol{H}} \cdot \underline{\boldsymbol{v}}\left(f_{d}\right)\right)^{2} d f_{d}$,

where the region of integration has to be appropriately chosen, and a Doppler-frequency dependent weighting of the integral may also be applied. In the multichannel resampling context, $P R F_{\text {mult } i}$ is for instance a meaningful choice.

Applying (18) for $\underline{\boldsymbol{v}}\left(f_{d}\right)$ defined by (12) and each $G_{G O A L}\left(f_{d}, k\right)$ in the output grid is a natural choice for (17). For each output grid sample index $k, 1 \leq k \leq N_{\text {out }}$, the solution to the optimum weights in the MSE sense can be obtained by minimizing (18) by means of its complex gradient [21]. This yields weights proportional to the cross-correlation between the goal pattern and the array manifold vector,

$\underline{\boldsymbol{\sigma}}_{G}[k] \triangleq \int G_{G O A L}^{*}\left(f_{d}, k\right) \cdot \underline{v}\left(f_{d}\right) d f_{d}$

weighted by the inverse of the array manifold's crosscorrelation matrix

$\underline{\boldsymbol{R}}_{v} \triangleq \int \underline{\boldsymbol{v}}\left(f_{d}\right) \cdot \underline{\boldsymbol{v}}\left(f_{d}\right)^{H} d f_{d}$

The set of optimum MSE weights is then given by 
$\underline{\boldsymbol{w}}_{M S E}[k]=\underline{\boldsymbol{R}}_{v}{ }^{-1} \cdot \underline{\boldsymbol{\sigma}}_{G}[k]$.

This solution has the desirable property of achieving the closest possible implementation of the desired set of output patterns. Let the power of the goal pattern be described by

$p_{G} \triangleq \int\left|G_{G O A L}\left(f_{d}\right)\right|^{2} d f_{d}$.

Then, using the definitions (19) and (20), (18) can also be written as

$\xi_{M S E}=\underline{\boldsymbol{w}}^{H} \cdot\left(\underline{\boldsymbol{R}}_{v} \cdot \underline{\boldsymbol{w}}-\underline{\boldsymbol{\sigma}}_{G}\right)-\underline{\boldsymbol{w}}^{T} \cdot \underline{\boldsymbol{\sigma}}_{G}^{*}+p_{G}$,

and the optimum MSE solution of (20) leads to

$\xi_{M S E_{\min }}=p_{\boldsymbol{G}}-\underline{\boldsymbol{\sigma}}_{G} \cdot \underline{\boldsymbol{R}}_{v}^{-1} \cdot \underline{\boldsymbol{\sigma}}_{G}^{H}$,

where the last term is a scalar and thus equal to its transpose and the $k$ (output grid sample index) dependence is omitted to simplify the notation, though it should be kept in mind.

The structure of (21), notably the dependence on $\underline{\boldsymbol{\sigma}}_{G}$, implies that the method automatically selects - from the physical channels in different positions during the pulse cycles - the elements with higher correlation to a particular output position. Even though the pulse separation induced baselines introduce, as expected, a notable decorrelation between the elements of the proposed extended manifold, no degradation ensues from the use of additional channels. Should they be too distant from the desired sample position and thus uncorrelated (leading to an element of $\underline{\boldsymbol{\sigma}}_{G}$ with low magnitude), the corresponding weights are accordingly very low in magnitude. The small gain avoids therefore a possible degradation from uncorrelated samples. In fact, (24) implies that the MSE corresponding to the best approximation (which is in general nonzero) is given by the goal pattern's power minus the norm of $\underline{\boldsymbol{\sigma}}_{G}$ weighted by $\underline{\boldsymbol{R}}_{v}^{-1}$. This means that extending the manifold (and thus getting a longer $\boldsymbol{\sigma}_{G}$ ) in principle cannot reduce the quality of the approximation, since it causes $\xi_{M S E_{\min }}$ to get smaller ${ }^{2}$. Clearly, the entries of $\boldsymbol{\sigma}_{G}$ show lower and lower magnitudes with increasing distance from the goal pattern's phase center position, leading to a saturation effect in the sense that additional elements start having little impact. The fact that for a staggered SAR system an overall oversampling in azimuth is expected, means that some correlation and therefore performance gain is however possible from the usage of neighboring pulses.

The method introduced in this section will be referred to as MSE-VBS, to emphasize the choice of the cost function, in the remainder of this paper. An alternative cost function is examined in the following section.

\footnotetext{
${ }^{2}$ This is true as long as the model (12) holds, though in practice the delay (phase ramp) is not an adequate representation for the relationship between arbitrarily distant pulses, as effects like range cell migration (RCM) and scene decorrelation come into play if the corresponding baseline is too large, resulting in an effective limitation of the feasible pulse neighborhood.
}

\section{F. Joint Mean Squared Error (MSE) and Signal-to-Noise Ratio (SNR) Cost Function: a MSE-SNR Compromise}

As was also true for the primary beam steering method in Section II.C., the spatial restriction of the reflector's surface illumination (cf. Fig. 3 (b)) and the corresponding broadening of the secondary patterns result in a lower gain in comparison to the feed element's patterns (cf. Fig. 8 for an example of $G_{i}\left(f_{d}\right)$ and $G_{\text {sum }}\left(f_{d}\right)$ ). Using only a part of the reflector's surface at a time is the price for achieving the phase center diversity, but it also means that the MSE-VBS method in Section II.D bears the inherent risk of degrading the SNR of the data.

Moreover, the manifold extension may lead to the use of several potentially poorly correlated channels, in turn yielding a beamformer with poor signal-to-noise ratio (SNR) gain in comparison to other alternatives, especially when obtaining samples within the gaps. To counter these effects, a normalized SNR measurement [21] with respect to white noise

$\xi_{S N R}=\frac{\underline{\boldsymbol{w}}^{H} \cdot \underline{\boldsymbol{R}}_{v} \cdot \underline{\boldsymbol{w}}}{\underline{\boldsymbol{w}}^{H} \cdot \underline{\boldsymbol{w}}}$

may be incorporated into the cost function. Using (20), one may write

$\xi_{S N R}=\frac{\int\left|\underline{\boldsymbol{w}^{H}} \cdot \underline{\boldsymbol{v}}\left(f_{d}\right)\right|^{2} d f_{d}}{\underline{\boldsymbol{w}}^{H} \cdot \underline{\boldsymbol{w}}}$,

which is equivalent to an average of the output pattern gain over the Doppler region in which the correlations are estimated. Considering a signal with a spectrum shaped as the antenna pattern after DBF, $\underline{\boldsymbol{w}}^{H} \cdot \underline{\boldsymbol{v}}\left(f_{d}\right)$, and a flat white noise spectrum, it is also clear that (26) represents a form of normalized SNR measurement. Denoting the elements of the $N_{\text {win }}$ by $N_{\text {win }}$ matrix $\underline{\boldsymbol{R}}_{v}$ by $r_{i j}$ and the elements of the $N_{w i n}$-element weight vector $\underline{\boldsymbol{w}}$ by $w_{i}$, one may also write

$\xi_{S N R}=\frac{\sum_{i=1}^{N_{\text {win }}} r_{i i} \cdot\left|w_{i}\right|^{2}+\sum_{j=1}^{N} \sum_{\substack{i=1 \\ j \neq i}}^{N} r_{j i} \cdot w_{i}^{*} \cdot w_{j}}{\left.\sum_{i=1}^{N_{w i n} \mid} w_{i}\right|^{2}}$,

a form in which some SNR properties of the manifold and pattern after beamforming may be highlighted. Recalling that $r_{i i}$ is proportional to the power in each manifold element and $r_{j i}$, $j \neq i$ is the cross-correlation between the manifold element's patterns, it is apparent that, for a given set of weights, a better SNR is obtained if the manifold elements are more correlated, whereas a completely uncorrelated manifold yields poor gain. Conversely, given a manifold, the solution to optimize the SNR involves activating the elements to the extent that the additional signal power brought by their self and cross-correlation outweighs the penalty for activating additional elements (more entries $w_{i}$ and thus a larger denominator). In contrast to (18)/(23), which as discussed in the previous section tends to always improve as more elements are added to the manifold (cf. (24)), (27) is seen to be adversely affected by the manifold extension beyond a certain correlation threshold. This also means it is reasonable to use a term proportional to this cost function in 
addition to (18)/(23), in order to restrict the choice of samples to be used, in the interest of improving the output SNR. Doing so leads to a joint MSE-SNR cost function of the form ${ }^{3}$

$\xi_{J}=(1-\alpha) \cdot \frac{\xi_{M S E}}{n_{M S E}}+\alpha \cdot \frac{n_{S N R}}{\xi_{S N R}}$

where $n_{M S E}$ and $n_{S N R}$ are normalization factors, which allow the MSE and SNR to be matched in terms of numerical values, and $\alpha$ is a design parameter in the interval $[0,1]^{4} . \alpha=0$ leads to the optimal MSE solution seen before, while increasing values up to $\alpha=1$ results in a limiting case where the goal pattern (sampling) is completely disregarded, since the full emphasis is on the SNR of the solution.

Since the MSE is directly linked to the regularity of the achieved output grid and irregularity translates into aliasing and residual azimuth ambiguities, the MSE may be viewed as a proxy for the residual ambiguity levels. Therefore the compromise being introduced is that of noise rejection on the one hand and ambiguity rejection on the other, which is a wellknown trade-off for other multichannel SAR system processing alternatives [7].

Applying the complex gradient operator to (28) leads to a nonlinear system of equations to determine the $N_{\text {win }}$ optimal complex weights, which nonetheless may be solved numerically using the closed form solutions available for the limiting cases $\alpha=0$ or $\alpha=1$ as first guesses, namely

$$
\begin{aligned}
& \frac{1-\alpha}{n_{M S E}} \cdot\left(\underline{\boldsymbol{R}}_{v} \cdot \underline{\boldsymbol{w}}-\underline{\boldsymbol{\sigma}}_{G}[k]\right)+\alpha \cdot n_{S N R} \cdot \\
& \frac{\left(\underline{\boldsymbol{w}}^{H} \cdot \underline{\boldsymbol{R}}_{\boldsymbol{v}} \cdot \underline{\boldsymbol{w}}\right) \cdot \underline{\boldsymbol{w}}-\left(\underline{\boldsymbol{w}}^{H} \cdot \underline{\boldsymbol{w}}\right) \cdot\left(\underline{\boldsymbol{R}}_{\boldsymbol{v}} \cdot \underline{\boldsymbol{w}}\right)}{\left(\underline{\boldsymbol{w}}^{H} \cdot \underline{\boldsymbol{R}}_{\boldsymbol{v}} \cdot \underline{\boldsymbol{w}}\right)^{2}}=\underline{\mathbf{0}} .
\end{aligned}
$$

A possible figure of merit for the SNR of the pattern achieved by means of arbitrary weights $\underline{\boldsymbol{w}}$ is given by the integral of the patterns' gain inside the processed bandwidth $B w_{\text {proc }}$, normalized by the same integral for a reference pattern. This reference is chosen here to be $G_{\text {sum }}\left(f_{d}\right)$, yielding the quantity

$\Phi_{S N R}(\underline{\boldsymbol{w}})=\frac{1}{k_{\text {sum }}} \cdot \frac{1}{\underline{\boldsymbol{w}}^{H} \cdot \underline{\boldsymbol{w}}} \cdot \int_{B w_{\text {proc }}}\left|\underline{\boldsymbol{w}}^{\boldsymbol{H}} \cdot \underline{\boldsymbol{v}}\left(f_{d}\right)\right|^{2} d f_{d}$,

where the normalization factor is given by

\footnotetext{
${ }^{3}$ A similar strategy is adopted in [7], though in a different optimization context.

${ }^{4}$ Numerically speaking only the ratio of the weights between the coefficients of the two cost functions in (28) matters for the solution, so that a single parameter would suffice. The separation into the three parameters is however preferred to enable a more obvious interpretation of the design goal of the algorithm.
}

$k_{\text {sum }}=\frac{1}{N_{c h}} \cdot \int_{B w_{\text {proc }}}\left|\sum_{i=1}^{N_{c h}} G_{i}\left(f_{d}\right)\right|^{2} d f_{d}$.

The method described in this section, whose goal is to introduce a SNR-MSE compromise, will be referred to, in short, as the SNR-VBS method, and can be interpreted as an extension that complements the MSE-VBS method.

The next section - in contrast to this and the previous one does not introduce a new cost function, but rather addresses the so far neglected issue of equalizing the performance over the output grid by introducing an iterative technique that may be applied regardless of the cost function.

\section{G. Iterative Pattern Synthesis: Accommodating Pattern Distortions to Equalize Performance over The Grid}

The optimality of the MSE method in the least-squares (cf. Section II.E) sense means that the implemented patterns are as close as possible to the goal patterns. Nonetheless, residual distortions occur as a rule, and the implemented patterns are imperfect approximations of the goals. As the procedure is repeated over all samples to form the output grid, owing to the irregularity of $t_{R X}[i]$, some pattern approximations are less successful than others. This is especially true for the output samples that span the Tx blockage-induced gaps, as these require larger shifts of the phase centers. Moreover, regardless of whether (18) or (28) are minimized, the optimization takes place using information from a single output sample at a time, thus the knowledge of the other desired outputs over the grid is not used in the design and their varying degree of success cannot be accounted for. Conceptually, means to use the information from other output samples in the grid to implement a given pattern $G_{G O A L}\left(f_{d}, k\right)$ in order to better equalize the performance over the output samples are thus desirable.

A simple way of doing this is to exploit the degree of freedom of choosing $G_{\text {common }}\left(f_{d}\right)$ in (14). As long as the phase relations regarding $t_{\text {out }}[k]$ hold, the output grid remains regular, and enforcing $G_{\text {common }}\left(f_{d}\right)=G_{\text {sum }}\left(f_{d}\right)$ is not strictly necessary, though physically meaningful for reflector systems. The common pattern can also be shaped such that the output grid is more readily implementable by the given input manifold, in the sense of improving the worst-case implementation. The incorporation of an average distortion to the common component of the design goals may lead to more readily achievable patterns without violating the regularity, the main objective of the resampling. Moreover, if the design is done iteratively, the information from the other patterns in the grid is readily available at the end of each iteration. One may thus propose the following logic for the common pattern design. The previous choice is maintained for the first iteration, i.e.,

$G_{\text {common }}^{0}\left(f_{d}\right)=G_{\text {sum }}\left(f_{d}\right)$

However, at iteration $i$, 
$G_{\text {common }}^{i+1}\left(f_{d}\right)=\frac{1}{N_{\text {win }}}$.

$\sum_{k=1}^{N_{\text {win }}}\left(\underline{\boldsymbol{w}}^{\boldsymbol{i}}[k]\right)^{\boldsymbol{H}} \cdot \underline{\boldsymbol{v}}\left(f_{d}\right) \cdot \exp \left(+j \cdot 2 \cdot \pi \cdot t_{\text {out }}[k] \cdot f_{d}\right)$,

where $\underline{w}^{i}[k]$ denotes the weights for the $k^{t h}$ pattern in the grid at the iteration under consideration and both the manifold and the weights have dimension $N_{\text {win }}$. This means that the mean common pattern effectively achieved by the implementation is calculated, and passed on as a less strict design goal to the next iteration. This allows lower MSEs to be achieved in the worst cases over the grid and thus improves the overall approximation. It should be noted that, if (32) and (33) are used in combination with (18), lower MSEs than those of the MSE-VBS method in Section II.D may be obtained because of the change in the design goal, hence without contradiction to the optimality of that method. A degradation of $G_{\text {common }}\left(f_{d}\right)$ in comparison to the initial sum pattern is possible, but the impact is small as long as the worst-case distortions are not excessive. The effect can be controlled by proper design of the PRI sequence.

A stop criterion for the iteration is needed, and one possibility is improvement of the average MSE over the grid from the current iteration to the previous. If (28) is used, it is also possible to use an average of the SNR figure of (30). In the latter case, a feedback of the parameter $\alpha$ into the design goal tends to enhance the emphasis on the SNR and improve the performance with this regard, though increasing the minimum achievable MSE and possibly slowing convergence to lower MSE values.

To conceptually sum up the methods discussed in this section, a flow chart of the inputs employed for the weight calculations is provided in Fig. 5, comparing the different alternatives described in the subsections therein.

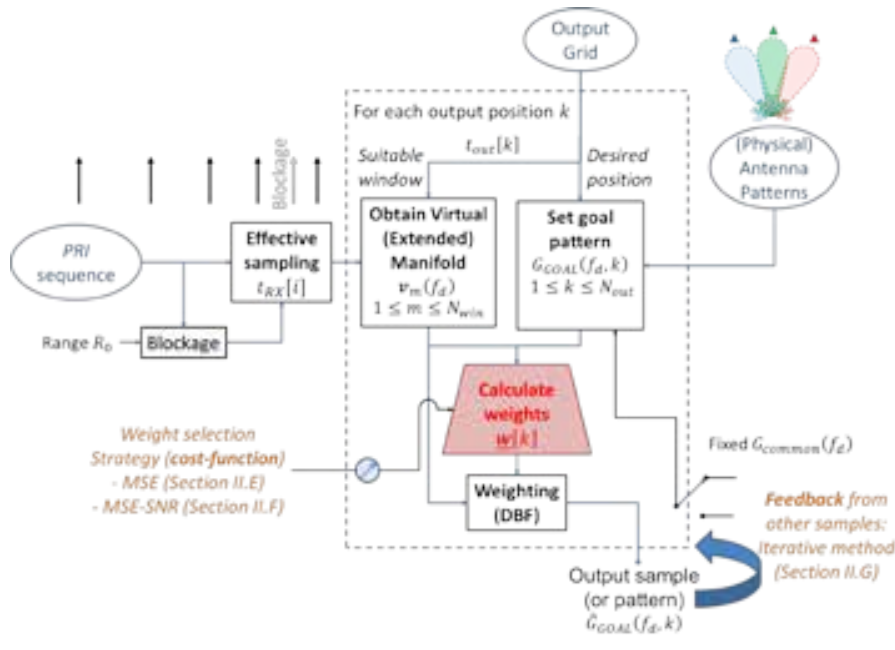

Fig. 5: Flow chart highlighting the information needed for the weight calculation. Knowledge of the antenna patterns and sampling conditions is common for all the variations of the VBS method (cf. Section II.D). The choice of the cost function (cf. Sections II.E and II.F) determines the weight calculation strategy, whereas the iterative method of Section II.G is a possibility to introduce feedback from other samples in the output grid into the weight calculation.

\section{ANALYSIS AND COMPARISON OF METHODS}

This section illustrates the application of the aforementioned methods and discusses some aspects of their performance. The structure is as follows: Section III.A provides the common background of the simulation scenario, Section III.B analyses the synthesis of a single pattern or output sample with varying sensitivity parameter $\alpha$ values, and, finally section III.C analyses the synthesis of the whole output grid required for a particular case of resampling.

\section{A. Description of Simulation Scenario}

In order to analyze and compare the performance of the different methods, an illustrative scenario is considered, taking as reference one of the high azimuth resolution modes of the Tandem-L mission proposal [1]. The goal is to image from an orbit height of $745 \mathrm{~km}$ a swath of $350 \mathrm{~km}$ on ground with $3.0 \mathrm{~m}$ azimuth resolution in L-band, using a parabolic reflector antenna architecture [8], as depicted in Fig. 6.
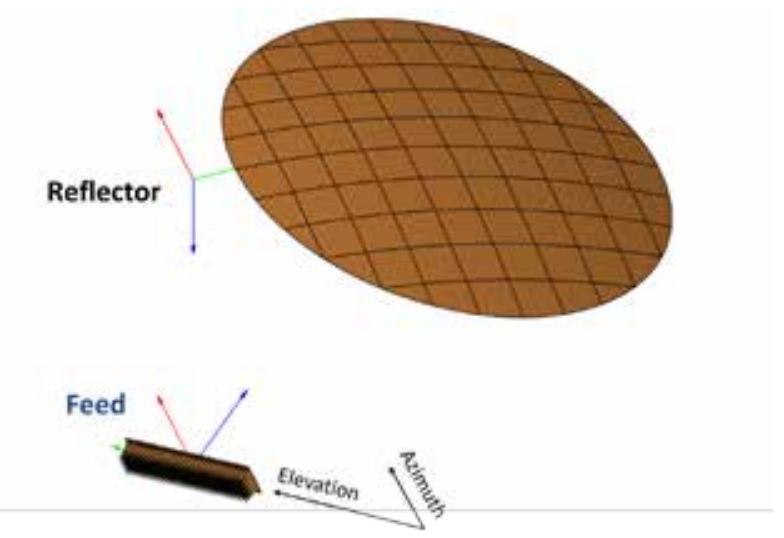

Fig. 6: Antenna system geometry highlighting multichannel feed and reflector rim. The feed system consists of 32 elements in elevation and 6 in azimuth, combined pairwise to form 3 azimuth channels.

The 3-m imaging mode is being considered as an option for implementation in Tandem- $\mathrm{L}$ (in addition to the 7-m imaging mode with one azimuth channel).

As an example, a simulated point target in the center of the scene at a ground range of $485 \mathrm{~km}$ is considered. A periodically non-uniformly sampled multichannel signal as discussed in Section II.A results from the use of a staggered PRI sequence on a system with 3 channels on receive in azimuth. In this case, the 3 channels are obtained by the pairwise combination of 6 azimuth feed elements spaced at $0.6 \lambda$ in azimuth. The relevant system parameters are summarized in TABLE I.

The description of the PRI cycle and the physical antenna patterns provides the basis for the characterization of the extended manifold used as input for the resampling. With respect to the former, timing analysis [15],[16] shows that for this particular range the 3rd and the 32nd pulse from the sequence of $N_{P R I}=33$ pulses are lost due to transmission events. This leads 
to an effective $N_{\text {eff }}=31$ number of pulses as shown in the sampling configuration in Fig. 7.

TABLE I

SiMULATION SCENARIO PARAMETERS

\begin{tabular}{lll}
\hline \multicolumn{3}{c}{ Platform and swath parameters } \\
\hline Quantity & Symbol & Value \\
\hline Orbit height & $h_{\text {orbit }}$ & $745 \mathrm{~km}$ \\
Swath width on ground & $W_{\text {ground }}$ & $350 \mathrm{~km}$ \\
Minimum/maximum look angle & $\theta_{\min } / \theta_{\max }$ & $23.4 / 40.9 \mathrm{deg}$ \\
\hline \hline
\end{tabular}

\begin{tabular}{|c|c|c|}
\hline \multicolumn{3}{|c|}{ Reflector and feed parameters } \\
\hline Quantity & Symbol & Value \\
\hline Diameter & $D$ & $15.0 \mathrm{~m}$ \\
\hline Focal length & $F$ & $13.5 \mathrm{~m}$ \\
\hline Feed offset in elevation & $d_{O F F}$ & $9.0 \mathrm{~m}$ \\
\hline Center frequency & $f_{0}$ & $1.2575 \mathrm{GHz}$ \\
\hline $\begin{array}{l}\text { Number of channels in } \\
\text { elevation/azimuth }\end{array}$ & $N_{e l} / N_{a z}$ & $32 / 3$ \\
\hline Channel spacing in elevation/azimuth & $d_{e l} / d_{a z}$ & $0.68 \lambda / 1.2 \lambda$ \\
\hline Elevation tilt angle w.r.t. nadir & $\theta_{\text {tilt }}$ & $32.4 \mathrm{deg}$ \\
\hline
\end{tabular}

\begin{tabular}{lll}
\hline & Pulse sequence parameters & \\
\hline Quantity & Symbol & Value \\
\hline Average PRF & $\overline{P R F}$ & $2700 \mathrm{~Hz}$ \\
Initial PRI & $P R I_{0}$ & $386 \mu \mathrm{s}$ \\
PRI sequence step & $\Delta$ & $-0.98 \mu \mathrm{s}$ \\
PRI sequence length & $N_{P R I}$ & 33 \\
Pulse length & $T_{P}$ & $14.8 \mu \mathrm{s}$ \\
Duty cycle & $T_{d c}$ & $4 \%$ \\
\hline \hline & & \\
& Processing parameters & \\
\hline Quantity & Symbol & Value \\
\hline Goal azimuth resolution & $\delta_{a z}$ & $3 \mathrm{~m}$ \\
Processed bandwidth & $B w_{\text {proc }}$ & $2494 \mathrm{~Hz}$ \\
Number of pulses in azimuth & $N_{p}$ & 31 \\
beamformer window & $R_{0}$ & $485 \mathrm{~km}$ \\
Target ground range & & \\
\hline \hline
\end{tabular}

In the next sections, the particular case of $N_{\text {win }}=N_{c h} \cdot N_{\text {eff }}$, meaning the window of inputs for formation of the output grid is chosen to be a cycle of the PRI sequence, is considered.

In turn, the magnitude of the far-field (secondary) antenna patterns $G_{i}\left(f_{d}\right)$ of the 3 azimuth channels, as well as the corresponding $G_{\text {sum }}\left(f_{d}\right)$, are shown in Fig. 8. The patterns were simulated using the software TICRA GRASP [22] and show no appreciable phase difference between the elements.

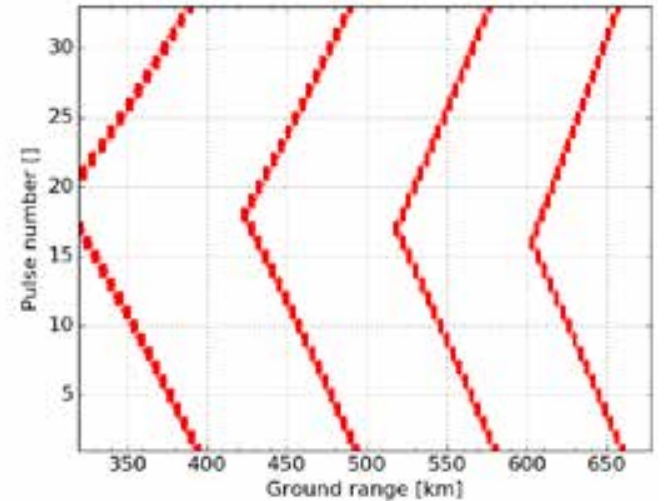

(a)

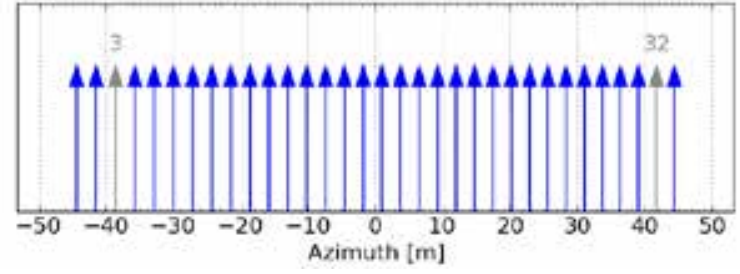

(b)

Fig. 7: Analysis of the transmit-event induced loss of pulses over range. (a) shows the blockage diagram for the whole swath, with ground range as abscissa and the pulse index (1-33) as ordinate. For each ground range, red boxes indicate the indices of the lost pulses, and the fact that two consecutive losses never occur for the considered ranges - a consequence of the sequence design criterion - can be observed. (b) depicts the azimuth sampling (in blue) and blocked pulses (in grey) over one PRI sequence cycle at the particular ground range of $485 \mathrm{~km}$, roughly the center of the swath.

As expected for an ideal reflector (cf. Section II.C), the phase centers coincide but the Doppler region covered by each element differs. The sum pattern (dashed line) is seen to be much broader, and its width is approximately given by the combined beamwidth of all elements.

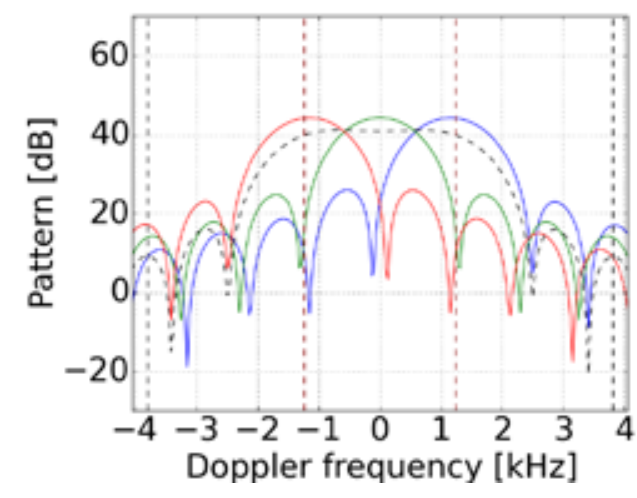

Fig. 8: Antenna pattern magnitude for the 3 Rx physical channels (solid lines) and corresponding sum pattern (dashed line). The outer vertical dashed lines highlights the limits of the effective multichannel $P R F_{\text {multi }}=7609 \mathrm{~Hz}$ and the inner ones the processed bandwidth $B w_{\text {proc }}$. 
B. Synthesis of a single Goal Pattern: The Impact of The SNR Sensitivity Parameter $\alpha$

As a first example, the synthesis of $G_{G O A L}\left(f_{d}, 0\right)$, i.e., the pattern corresponding to the first sample of the output grid is considered. Fig. 9 shows the sampling configuration of the extended manifold as well as the desired phase center position. Due to the assumption of $N_{\text {win }}=N_{c h} \cdot N_{e f f}$, the extended manifold has $N_{\text {manifold }}=93$ elements (represented by blue circles), corresponding to the physical channels over the whole cycle of received pulses (shown as arrows). The output regular grid (represented through an 'x') has as well $N_{\text {out }}=93$ samples over a PRI cycle. The first output sample is highlighted in red. In this case, a shift of $-0.65 \mathrm{~m}$ is required with respect to the position of the nearest pulse, namely, the first one of the cycle.

In order to illustrate the performance of the methods in Sections II.D and II.F, as well as to provide a better understanding of the inherent MSE-SNR compromise, the implementation of this particular pattern with varying values of the sensitivity parameter $\alpha$ in the interval $[0,1]$ is considered in the following. The joint cost function (28) is used with the following parameters: $n_{M S E}$ is taken to be the power of the sum pattern $G_{\text {sum }}\left(f_{d}\right)$ given by (16) and $n_{S N R}$ is equal to the SNR figure of the same sum pattern $k_{\text {sum }}$ of (31) divided by 100 . This choice of parameter values is motivated by the fact that the normalized MSE figure for this example was found to be in the order of -20 to -30 dB, while the normalized SNR figure $\Phi_{S N R}$ (cf. (30), (31)) is in the order of 0.0 to $-10 \mathrm{~dB}$, and hence the factor 100 was chosen to better match numerically the values of the two figures and thus adjust the sensitivity to $\alpha$. The correlations between the manifold elements, as well as the MSE measurements (cf. (18), (19), (20)) are done by integration over PRF $F_{\text {multi }}$.

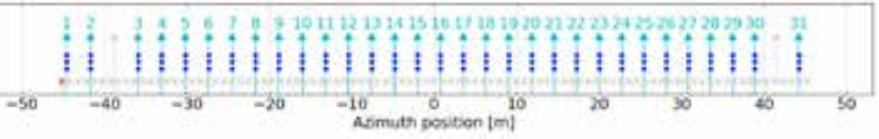

(a)

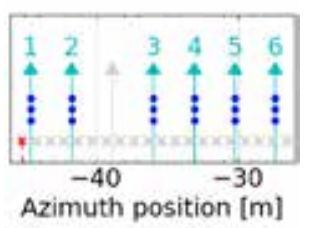

(b)

Fig. 9: Sampling configuration for extended manifold and desired regular grid. Arrows represent the received pulse position, highlighting the 31 available pulses. The blue circles represent the phase center position of the samples from the different azimuth channels. The desired regular grid positions are represented by an " $\mathrm{x}$ ", and the particular phase center position for the pattern under analysis is highlighted in red. (a) shows the configuration for the whole PRI sequence and (b) a zoom around the first gap.

Firstly, Fig. 10 shows examples of the achieved patterns for five equally spaced values of $\alpha$ in the sweep from 0.0 to 1.0 . The patterns are represented in terms of gain and phase error, the latter with respect to the phase ramp dictated by the required phase center position $t_{\text {out }}[0]$. It should be highlighted that the gain mentioned here refers to the usual definition [21], i.e.

$Q\left(f_{d}, \alpha\right)=\frac{\left\|\underline{\boldsymbol{w}}(\alpha)^{H} \cdot \underline{\boldsymbol{v}}\left(f_{d}\right)\right\|^{2}}{\underline{\boldsymbol{w}}(\alpha)^{H} \cdot \underline{\boldsymbol{w}}(\alpha)}$,

and should not be confused with the pattern approximation $\widehat{G}_{G O A L}\left(f_{d}, \alpha\right)=\underline{\boldsymbol{w}}(\alpha)^{\boldsymbol{H}} \cdot \underline{\boldsymbol{v}}\left(f_{d}\right)$ itself. This distinction is important in this context as the pattern (here understood as the mere linear combination, without normalization of the weight magnitude) influences the MSE, while the gain in (34) influences the SNR.

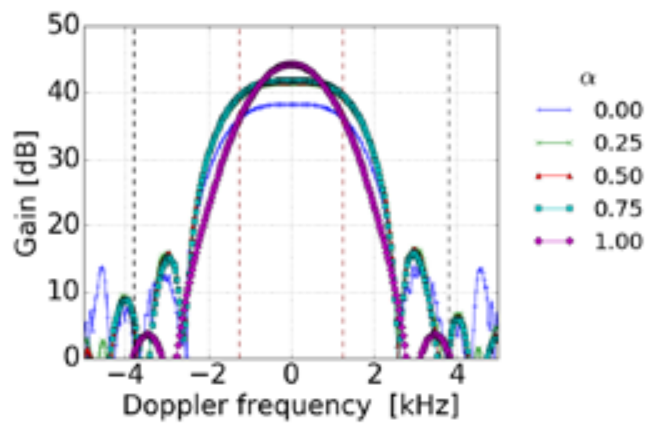

(a)

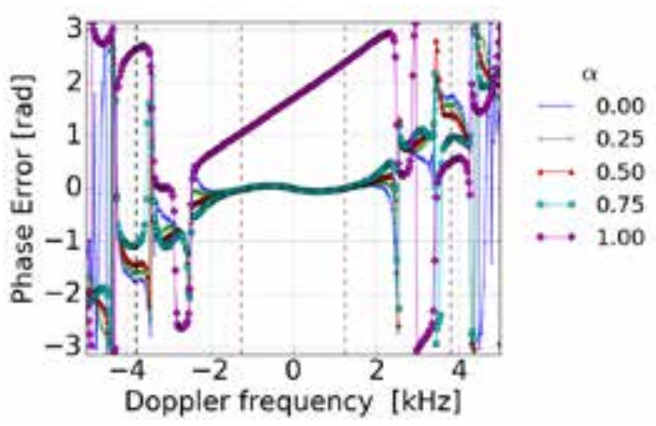

(b)

Fig. 10: Analysis of achieved patterns $\widehat{G}_{G O A L}\left(f_{d}, \alpha\right)=\underline{\boldsymbol{w}}(\alpha)^{\boldsymbol{H}} \cdot \underline{\boldsymbol{v}}\left(f_{d}\right)$ as a function of $\alpha$, in terms of gain and phase. (a) shows the pattern's gain and (b) the error with respect to the desired phase center position. The inner and outer dashed lines delimitate $B w_{\text {proc }}$ and $P R F_{\text {multi }}$, respectively.

As discussed in Section II.F, increasing values of $\alpha$ increase the importance of the SNR component in the joint cost function (cf. (28)). Correspondently, it can be seen from the plots that, as $\alpha$ increases, the pattern's gain also does, reflecting an improved SNR. Conversely, the phase errors are also seen to increase, indicating an MSE worsening. The shape of most patterns in Fig. 10 (a) is nonetheless seen to be stable and resemble very closely that of $G_{s u m}\left(f_{d}\right)$, as visible in the figure. An exception occurs for $\alpha=1.0$, a case of theoretical interest leading to the best achievable SNR without actually implementing any resampling. In the latter case, the shape of the pattern is unrelated to the previous one. Another interesting aspect is the considerable change in the gain when $\alpha$ changes from 0.0 to 0.25 , even 
though neither the shape of the pattern nor the phase error changes abruptly. This is an indication of the non-linear behavior of (29), which the few examples in Fig. 10 do not fully allow to characterize.

A more complete characterization follows from the analysis in Fig. 11, where the normalized MSE (with respect to $n_{M S E}$ ) and $\Phi_{S N R}$ of the achieved approximation $\widehat{G}_{G O A L}\left(f_{d}, \alpha\right)$ are plotted against the value of $\alpha$ for 400 values within the interval $[0,1]$.

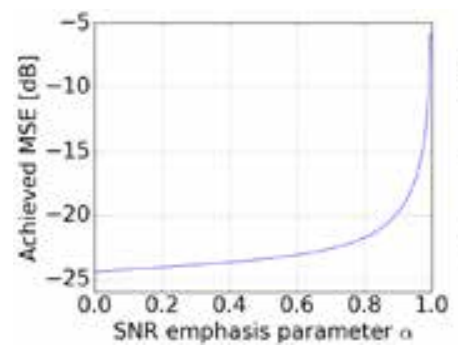

(a)

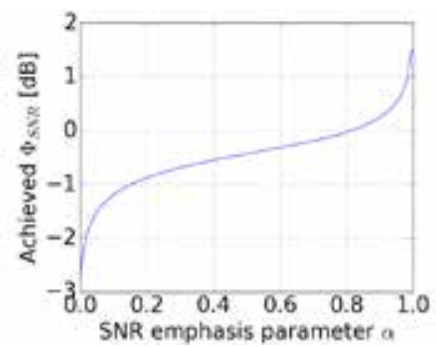

(b)
Fig. 11: Analysis of achieved patterns $\hat{G}_{G O A L}\left(f_{d}, \alpha\right)=\underline{\boldsymbol{w}}(\alpha)^{\boldsymbol{H}} \cdot \underline{\boldsymbol{v}}\left(f_{d}\right)$ as a function of $\alpha$ in terms of normalized MSE and SNR figures. (a) shows the pattern's MSE with respect to the goal pattern, normalized to the power of the sum pattern and (b) the SNR figure $\Phi_{S N R}$.

As expected, both the MSE and the SNR increase with increasing values of $\alpha$, highlighting the aforementioned trade-off between the two parameters. It is nonetheless interesting to note that the sensitivity of the two curves is different, in another indication of non-linear behavior. The MSE varies slowly with $\alpha$ up to circa 0.8 and then increases abruptly, indicating the increasing disregard of the sampling conditions by the cost function. On the other hand, the SNR increases quickly with $\alpha$ for values up to 0.2 , remains fairly stable up to 0.8 and then quickly increases again up to the optimum value. This behavior also explains what was visualized in Fig. 10: the change from 0.0 to 0.25 kept the pattern shape and phase errors (and hence the MSE) fairly constant while causing a visible difference in the gain (and hence the SNR); the changes from 0.25 up to 0.75 had visually little effect in both regards; and the final change to 1.0 led to a high-gain pattern (the best SNR figure) which is however completely different from the goal and shows a correspondingly high phase error (hence the high MSE).

The numerical values of the boundaries of the $\alpha$ regions clearly depend on the normalization parameters $n_{M S E}$ and $n_{S N R}$, which adjust the sensitivity of the cost function to $\alpha$. However, as the extreme cases do not change, a change in these parameters represents a mere scaling of the curve with respect to the abscissa values, not changing the general behavior. In terms of pattern design, the interesting point is that a considerable increase in the SNR can be achieved without a great degradation of the MSE by increasing $\alpha$ up to certain threshold. In fact, the optimum MSE solution incurs a relatively high SNR penalty by using all possible extended manifold elements including fairly uncorrelated elements (corresponding to distant pulses), which do not contribute to a great improvement of the MSE, while degrading the SNR considerably. With moderately low values of $\alpha$, very similar patterns are achieved with a lower weight magnitude, what can be interpreted as a better distribution of the activation energy, made possible by disregarding these fairly uncorrelated extended manifold elements.

\section{Synthesis of Full Output Grid: Comparison between Methods}

In this section, the achieved patterns are analyzed over the whole output grid, to complete the resampling process and achieve the final goal of the method.

First of all, the MSE-VBS method described in Section II.E (or equivalently the SNR-VBS one of Section II.F with $\alpha=0$ ) is considered as a solution to the resampling problem, so as to assess the closest possible implementation of the patterns. Evaluating the optimal MSE weights for every sample using (14) and (15) with $G_{\text {common }}\left(f_{d}\right)=G_{\text {sum }}\left(f_{d}\right)$ (cf. (16)) leads to the 93 patterns depicted in Fig. 12 in terms of power and phase error, i.e., after removal of the goal phase ramp of (14), (15) independently for each of the output samples. The similarity between the amplitude of the patterns in Fig. 12 and the sum pattern in Fig. 8 is clear, indicating an implementation close to the desired patterns. Within the main beam, the patterns show stable magnitudes and very low residual phase errors with respect to the desired phase center positions, indicating that successful regularization was achieved over the grid.

Given the advantages of using the SNR-VBS method (cf. Section III.B), the implementation of the grid using (28) with $\alpha=0.6$ is also considered, both directly and with the addition of the iterative method explained in Section II.G.

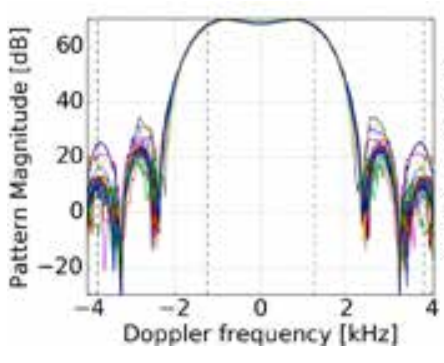

(a)

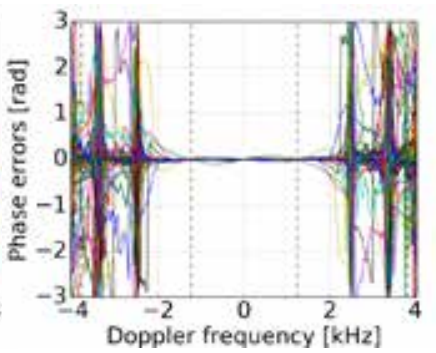

(b)
Fig. 12: Set of output patterns obtained from the optimal MSE method. (a) depicts pattern magnitude and (b) phase after removal of the sample-specific linear phase ramp (cf. (14) and (15)), highlighting residual phase errors with respect to ideal regular sampling. $B w_{\text {proc }}$ and $P R F_{\text {multi }}$ are marked by red and black dashed lines, respectively, in both plots.

The normalized MSE (with respect to $n_{M S E}$, the power of $G_{\text {sum }}\left(f_{d}\right)$ ) and the SNR scaling $\Phi_{S N R}(\underline{w})$ of (30) over the output patterns are shown for the mentioned methods in Fig. 13. The plots on the top refer to the MSE-VBS method of Section II.D. The ones in the middle were obtained with the SNR-VBS method of Section II.F, evaluated with $\alpha=0.6$. The plots on the 
bottom show the results for the iterative method of Section II.G, again using (28) with $\alpha=0.6$.
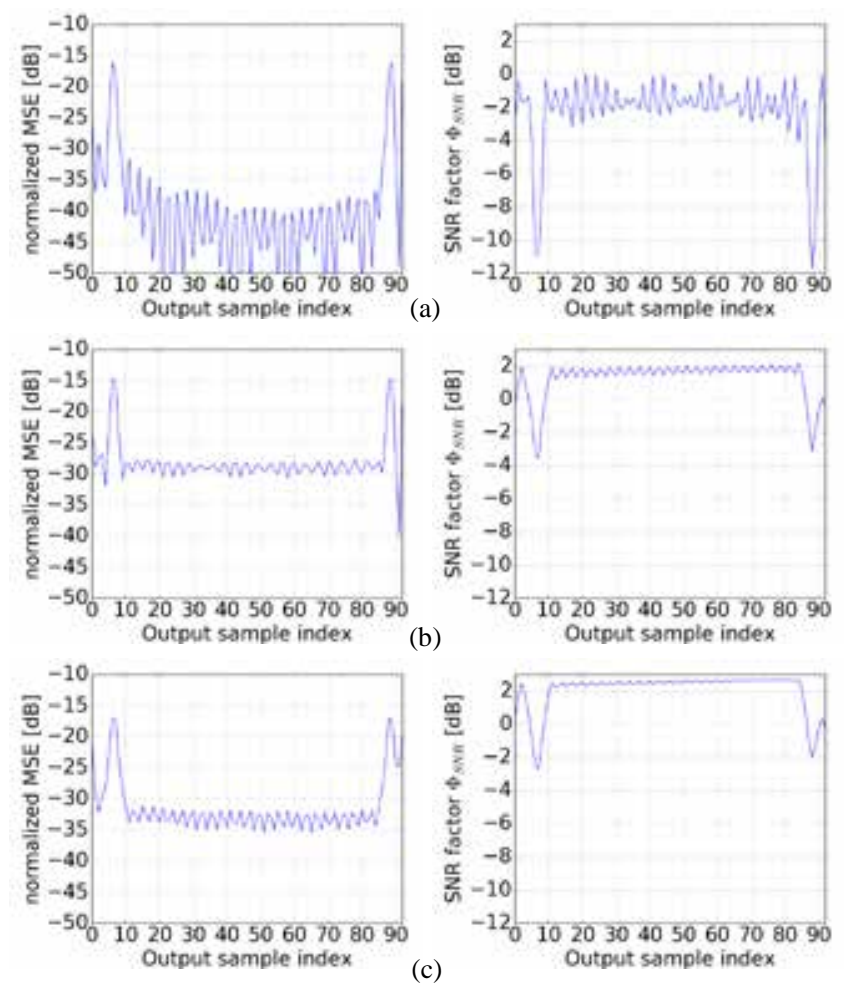

Fig. 13: Normalized MSE and $\Phi_{S N R}$ over output patterns/samples for different methods: (a) shows results for the MSE-VBS, (b) for the non-iterative SNR-VBS and (c) for the iterative SNR-VBS LS regularization methods. For the last two methods, $\alpha=0.6$ was adopted.

A comparison of the results on the top and in the middle of Fig. 13 highlights once again the compromise between the MSE and the SNR as shown in Section III.B, embodied by the design parameter $\alpha$. Introducing the iterative procedure ((b) vs. (c)) enhances, on average, both MSE and SNR, with a larger improvement for the worst cases, as was the goal. The ripple in $\Phi_{S N R}$ over the samples is also reduced, indicating that a more uniform performance was achieved. In all cases, the performance for the samples within the region of the blockage-induced gaps is clearly worse. This is expected and due to the larger phase center shift with respect to the input grid required to fill those gaps.

As a final illustration of the method's characteristics, the actual illumination of the reflector in each position corresponding to the received pulse is plotted in Fig. 14. The abscissa of the sub-plots corresponds to azimuth in meters, and each sub-plot consists of an illustration of the sampling configuration of a particular output sample (bottom) and the corresponding reflector surface illumination (top). In the top plots, the projection of the reflector surface and its illumination is represented for each of the 31 available pulses at the corresponding Rx positions over azimuth. At each pulse position, the physical channels are combined according to the weights of the MSE-SNR method with $\alpha=0.6$, and the color coding indicates the resulting power levels.
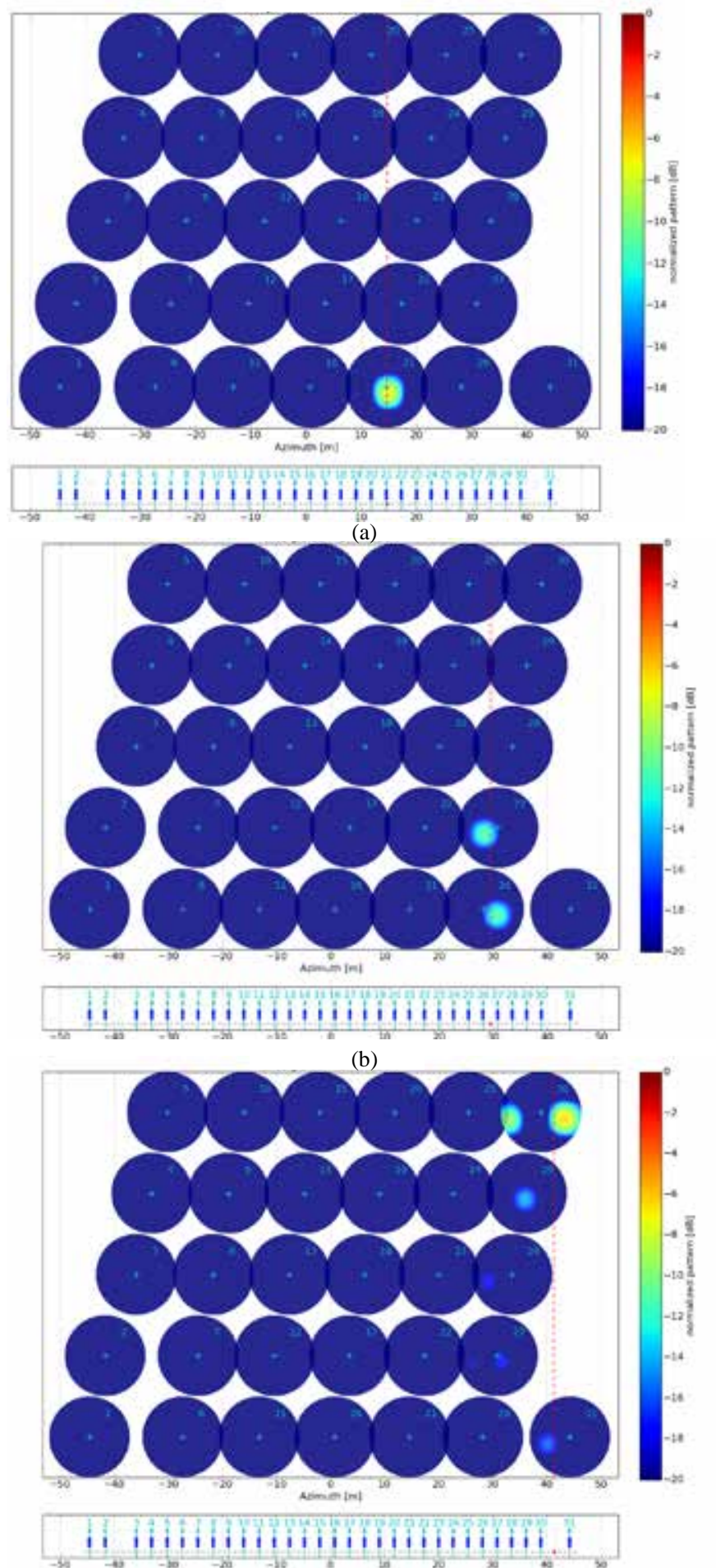

(c)

Fig. 14: Illustration of reflector illumination. (a) Output sample of index 61, a case in which the phase center shift is minor and no great contribution from neighboring pulses is observed. (b) Output sample of index 76, as an illustration of a case in which mainly two neighboring pulses are used to form the output pattern. (c) Output sample of index 88, which occurs during the second Tx-event induced gap, showing how several pulses are combined, which degrades the SNR. 
Note that each output sample's pattern is a result of the sum of the contributions of all pulses, even though the illumination is represented separately for each pulse. The vertical red dashed line indicates the desired output sample phase center position and the azimuth sampling as in Fig. 9 is represented below for reference. The figure contains three cases showing distinct behavior regarding the contribution of the neighboring pulses.

\section{Simulation Results}

The last section has addressed extensively the patterns of each sample in the regularly sampled output grid, to allow a better understanding of the introduced methods and of the compromises involved. In this section, focus is turned to the SAR performance achieved by them. Section IV.A continues the example described in Section III.A for a particular range, considering now the effect of the resampling on the simulated SAR data. Section IV.B in contrast introduces a new system design and analyses its SAR performance over the whole swath.

\section{A. Impulse Response Function (IRF) Analysis}

This subsection provides an Impulse Response (IR) analysis for the output of the VBS methods, with the parameters described in TABLE I.

Fig. 15 shows the magnitude of the simulated azimuth raw data after resampling with the optimal MSE weights. Fig. 15 (a) depicts the channel's magnitude in time domain plotted against instantaneous Doppler, and its shape shows an ensemble of the patterns seen in Fig. 12. The simulation is noise-free, yet the sidelobe regions appear to be noisy, an effect which is caused by the fast residual variation of the patterns between samples, being more pronounced in this region. This variation is more clearly visualized in Fig. 15 (b), where three cycles of the output grid are seen, starting from the center of the regularized channel. The abscissas represent sample indices, to emphasize the $N_{\text {out }}=93$ sample periodicity of the residual modulation.

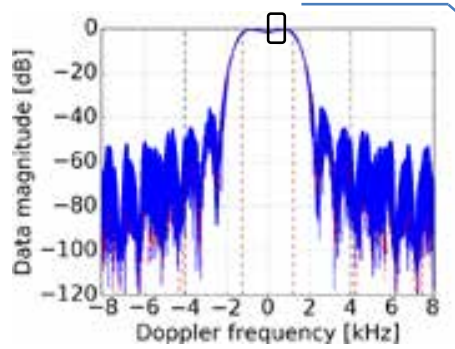

(a)

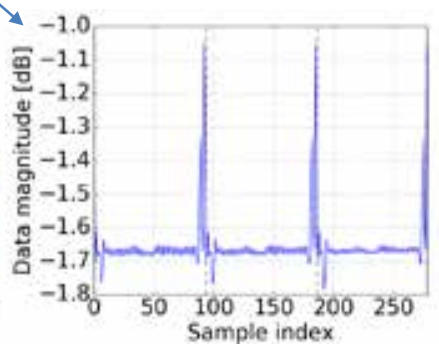

(b)
Fig. 15: Magnitude of raw data after resampling with the optimal MSE weights. (a) shows the whole resampled channel in time domain against instantaneous Doppler frequency (solid blue line) and the reference pattern (dashed red line); (b) a zoom over three cycles of 93 samples each, starting from the center of the regularized channel, to highlight the residual modulation of the resampled signal. The relevant frequency regions $B w_{\text {proc }}$ and $P R F_{\text {multi }}$ are marked by red and black dashed lines, respectively, in the left plot.

The sample indices are those of the resampled channel's time axis, sampled at $P R F_{\text {multi }}=7609 \mathrm{~Hz}$. In Fig. 15 (a), the instantaneous Doppler frequency of the target is calculated from the geometry for each of the corresponding time instants. The zoom (with respect to the ordinates) is taken starting from zero Doppler, a region where this effect is seen to be small, as is the case over the main beam and in particular over the processed bandwidth, of greater importance to the final focused signal quality. The relevance of the effect in the sidelobe region and especially outside the multichannel PRF area is reduced by the low gain levels of this part of the signal, which contributes mostly to residual azimuth ambiguities.

The focused impulse responses of the data regularized by the MSE-VBS and non-iterative SNR-VBS methods are plotted in Fig. 16, versus the instantaneous Doppler frequency. The IR for the iterative SNR-VBS is not shown due to its similarity to the other plots.

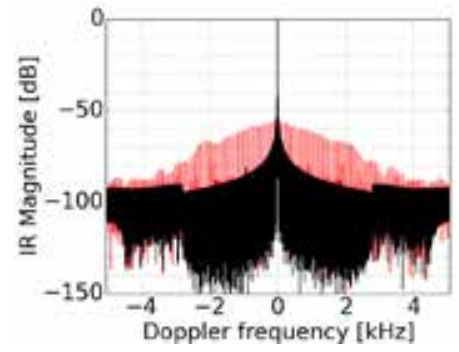

(a)

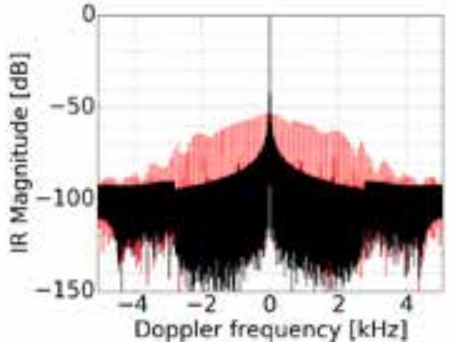

(b)
Fig. 16: Impulse responses of regularized data (red) and alias-free reference regularly sampled at $P R F_{\text {multi }}$ (black), for the MSE-VBS method (a) and (noniterative) SNR-VBS method (b).

Several azimuth ambiguities are seen in the impulse responses of the regularized data. Their peak levels are nonetheless very low, indicating successful application of the methods for resampling. The ambiguities occur at multiples of $\overline{P R F}_{\text {eff }} / N_{\text {eff }}$, as a result of residual regularization errors. It should be noted that $\overline{P R F}_{\text {eff }} /$ $N_{e f f}=1 / T_{P R I}$ is the rate at which the PRI sequence repeats itself, and that the residual deviations between the achieved patterns and the ideal $G_{G O A L}\left(f_{d}, k\right)$ lead to a periodical modulation of the samples in the output channel at this rate.

Figures of merit for the regularizations' output patterns and the impulse responses are summarized in TABLE II. There, $\delta_{A Z}$ is the 3-dB azimuth resolution, $A A_{P E A K}$ and AASR describe respectively peak and total azimuth ambiguity levels, while $\overline{M S E}$ and $\overline{\Phi_{S N R}}$ are averages (taken in linear units and then converted to $\mathrm{dB}$ ) of the quantities in Fig. 13. The estimation of the AASR in staggered SAR is addressed in [25] in detail.

The resolution goal of $3.0 \mathrm{~m}$ is achieved and acceptably low AASR levels are obtained for all methods. Furthermore, the proposed joint optimization (SNR-VBS) is seen to allow a considerable gain in SNR at the expense of an acceptably small loss in MSE and AASR levels. Since the design goal of (18) is to enforce regularity, the MSE and AASR levels are directly linked and the MSE-SNR compromise translates into an AASR-SNR one. It should, however, be noted that $G_{\text {common }}\left(f_{d}\right)$ also affects the final MSE levels, and the change in this parameter between 
the two last methods (non-iterative and iterative SNR-VBS) is the reason why the iterative method achieved a slightly worse AASR despite better $\overline{M S E}$.

TABLE II

COMPARISON BETWEEN LS METHODS IN TERMS OF ACHIEVED PATTERN AND IMPULSE RESPONSE FIGURES OF MERIT

\begin{tabular}{cccc}
\hline \hline & \multicolumn{3}{c}{ METHOD } \\
\cline { 2 - 4 } $\begin{array}{c}\text { FIGURE OF } \\
\text { MERIT } \\
\text { SYMBOL [UNIT] }\end{array}$ & $\begin{array}{c}\text { MSE-VBS } \\
(\alpha=0.0)\end{array}$ & $\begin{array}{c}\text { SNR-VBS } \\
(\alpha=0.6)\end{array}$ & $\begin{array}{c}\text { ITERATIVE SNR-VBS } \\
(\alpha=0.6)\end{array}$ \\
\hline$\delta_{A Z}[\mathrm{~m}]$ & 2.4 & 2.4 & 2.4 \\
$A A_{P E A K}[\mathrm{~dB}]$ & -55.3 & -53.1 & -53.3 \\
$\mathrm{AASR}[\mathrm{dB}]$ & -40.2 & -37.3 & $-36.8^{5}$ \\
$\overline{M S E}[\mathrm{~dB}]$ & -28.9 & -25.4 & -27.6 \\
$\bar{\Phi}_{S N R}[\mathrm{~dB}]$ & -1.9 & 1.4 & 2.2 \\
\hline \hline
\end{tabular}

As a reference, $\Phi_{S N R}$ for a frequency-adaptive MVDR beam [8] yields $3.2 \mathrm{~dB}$ for regular sampling. This technique requires Doppler-dependent weights and cannot directly be implemented without a regularly sampled input, but may be employed as an SNR upper bound. The proximity of the levels indicates that the performance achieved by means of the joint optimization is also satisfactory with regard to noise rejection, as intended.

\section{B. Performance Simulation over Swath}

Sections III and IV.A have provided an analysis of the synthesis of a single pattern and then of all the required patterns over the output grid, taking into account the sampling conditions imposed by a particular range. The goal of this section is in contrast to provide first-order designs for a fully polarimetric HRWS system in L-band and assess the achievable performance over a whole swath. For this, a reflector antenna system operated in a very fine $2.0 \mathrm{~m}$ resolution mode is considered, with a swath width of $400 \mathrm{~km}$, enough to provide global coverage in 7 days. Note that this system is different from the one presented in TABLE I and presents more challenging HRWS requirements.

In order to image the swath in quad-pol, the pulses with $\mathrm{H}$ and $\mathrm{V}$ polarization are interleaved on transmission and received simultaneously, and the sequence design follows the approach of [15], meaning the design is performed for a reference single-pol case with half of the mean PRI and then each PRI in the sequence is repeated twice for the interleaved dual-pol transmission sequence.

The interleaved polarization transmission has two noteworthy effects. First, with regard to the azimuth sampling, the spacing of the $\mathrm{V}$ (assumed to be the first polarization in the sequence) and the $\mathrm{H}$ transmitted pulses differs (namely by $\Delta$ between corresponding pulses), making the azimuth performance notably the AASR levels - dependent on the transmit polarization. Second, the signal is affected by both co-pol (even order) and cross-pol (odd order) range ambiguous returns, with a spacing corresponding to $2 \cdot \overline{P R F}$, which degrades the range ambiguity performance in comparison to an equivalent single-

\footnotetext{
${ }^{5}$ The low AASR levels indicate even a higher azimuth bandwidth could be processed, leading to a better resolution.
}

pol case operated at the same $\overline{P R F}$, making the quad-pol operation over wide swaths particularly challenging. The crosspol returns show closer proximity in comparison to the co-pol returns. This means that the former tend to dominate the range ambiguity-to-signal ratio (RASR) performance, unless the backscatter levels in cross-pol are much lower than in co-pol and compensate for the differences in range. Considering the L-band backscatter model of [26], the proximity effect is indeed dominant, which leads the cross-pol range ambiguity levels to be the design driver.

The design guideline is therefore to keep $\overline{P R F}$ as low as possible to counter range ambiguities. The lower $\overline{P R F}$ tends to degrade azimuth performance, but this is compensated in the antenna design. The antenna system and the mode's operational characteristics are described in TABLE III. The most relevant design modification with impact on the azimuth performance in comparison to the system of TABLE I is the reduction of the channel spacing and the usage of a larger reflector.

TABLE III

QUAD-POL SiMULATION SCENARIO PARAMETERS

\begin{tabular}{|c|c|c|}
\hline \multicolumn{3}{|c|}{ Platform and swath parameters } \\
\hline Quantity & Symbol & Value \\
\hline Orbit height & $h_{\text {orbit }}$ & $700 \mathrm{~km}$ \\
\hline Swath width on ground & $W_{\text {ground }}$ & $400 \mathrm{~km}$ \\
\hline Swath minimum/maximum look angle & $\theta_{\min } / \theta_{\max }$ & $22.0 / 42.8 \mathrm{deg}$ \\
\hline \multicolumn{3}{|c|}{ Reflector and feed parameters } \\
\hline Quantity & Symbol & Value \\
\hline Diameter & $D$ & $18.0 \mathrm{~m}$ \\
\hline Focal length & $F$ & $18.0 \mathrm{~m}$ \\
\hline Feed offset in elevation & $d_{O F F}$ & $12.0 \mathrm{~m}$ \\
\hline Center frequency & $f_{0}$ & $1.2575 \mathrm{GHz}$ \\
\hline $\begin{array}{l}\text { Number of channels in } \\
\text { elevation/azimuth }\end{array}$ & $N_{e l} / N_{a z}$ & $65 / 6$ \\
\hline Channel spacing in elevation/azimuth & $d_{e l} / d_{a z}$ & $0.6 \lambda / 0.8 \lambda$ \\
\hline Elevation tilt angle & $\theta_{\text {tilt }}$ & 34.3 deg \\
\hline Feed ohmic losses & $L_{\Omega}$ & $2.0 \mathrm{~dB}$ \\
\hline \multicolumn{3}{|c|}{ Pulse and Tx/Rx hardware parameters } \\
\hline Quantity & Symbol & Value \\
\hline Average PRF (both polarizations) & $\overline{P R F}$ & $2 \times 1750 \mathrm{~Hz}$ \\
\hline Initial PRI & $P R I_{0}$ & $313 \mu \mathrm{s}$ \\
\hline $\begin{array}{l}\text { PRI sequence step (between pulses of } \\
\text { same polarization) }\end{array}$ & $\Delta$ & $-1.34 \mu \mathrm{s}$ \\
\hline PRI sequence length & $N_{P R I}$ & $2 \times 41$ \\
\hline Pulse length & $T_{P}$ & $22.9 \mu \mathrm{s}$ \\
\hline Duty cycle & $T_{d c}$ & $2 \times 4 \%$ \\
\hline Pulse (chirp) bandwidth & $B w_{\text {chirp }}$ & $85 \mathrm{MHz}$ \\
\hline $\begin{array}{l}\text { Peak transmit power of a Transmit- } \\
\text { Receive Module (TRM) }\end{array}$ & $P_{T X}$ & $25.0 \mathrm{~W}$ \\
\hline Average transmit power & $\bar{P}_{T X}$ & $780.0 \mathrm{~W}$ \\
\hline System noise temperature & $T_{\text {noise }}$ & $649 \mathrm{~K}$ \\
\hline Transmitted polarizations & - & $\begin{array}{l}\mathrm{H}, \mathrm{V} \\
\text { (interleaved) }\end{array}$ \\
\hline
\end{tabular}


Processing parameters

\begin{tabular}{lll}
\hline Quantity & Symbol & Value \\
\hline Goal azimuth resolution & $\delta_{a z}$ & $2 \mathrm{~m}$ \\
$\begin{array}{l}\text { Processed bandwidth } \\
\text { Number of simultaneous elevation }\end{array}$ & $B w_{\text {proc }}$ & $3752 \mathrm{~Hz}$ \\
beams & $N_{\text {beams }}$ & 6 \\
$\begin{array}{l}\text { Elevation beamforming sidelobe } \\
\text { constraint }\end{array}$ & $\epsilon_{\text {SIDELOBE }}$ & $-40 \mathrm{~dB}$ \\
$\begin{array}{l}\text { Number of pulses in azimuth } \\
\text { beamformer window } \\
\text { SNR emphasis parameter }\end{array}$ & $N_{p}$ & 81 \\
\hline \hline
\end{tabular}

The smaller spacing improves the steering capabilities of the feed array and increases the grating-lobe free steering region for the primary beam; whereas the larger reflector increases the maximum possible phase center shift (cf. Section II.C). As a consequence, the performance of the resampling (operated by illuminating different areas of the reflector) is improved and a reduction of the mean PRF without severely impairing the azimuth performance levels achieved in the previous configuration is made possible. Another advantage of the large reflector is the large aperture area, which boosts antenna gain and improves the SNR for the same transmitted power. In light of this fact, the MSE-VBS is chosen as azimuth beamformer, shifting the AASR-SNR compromise in favor of improved AASR.

The DBF processing of the data starts over the elevation channels, with the goal of forming a high gain SCORE beam for each range. In practice, this assumption requires time-varying weighing of the channels to follow the echoes on ground and in the case of reflectors requires adaptive beamforming techniques, with knowledge of the antenna patterns, as e.g. the MVDR beamformer [8]. Furthermore, up to 6 simultaneous elevation beams are assumed.

Even though elevation beamforming is not the focus of this paper, it should be noted that the choice of the technique is crucial for the performance in terms of the range ambiguities and the signal-to-noise ratio. To simultaneously achieve the demanding performance levels required, the technique of [23] is employed, meaning the weights are result of an optimization to maximize the gain in the direction of the signal of interest (as in the MVDR case) while simultaneously imposing a sidelobe level constraint over the region over which the range ambiguities arise, resulting in a broad minimum $(40 \mathrm{~dB}$ below the beam maximum) over a grid of elevation angles. This elevation beamforming technique is explained in [24] in more detail. At each range, 11 of the 65 elevation elements are combined to form the SCORE beams. For the RASR calculations, the model of range ambiguities for staggered SAR discussed in [25] and the L-band backscatter model of [26] are employed.

Alongside the AASR and RASR, a key performance parameter for the mode is the noise equivalent sigma zero (NESZ). Estimation of the NESZ for multichannel systems takes into account the geometry, antenna patterns and RF hardware parameters, but also requires some special considerations [28]. The estimation results shown here are based on a single-channel system with the sum pattern (16) and the multichannel PRF, augmented by the SNR scaling factor calculated as an average over the output grid, as in (30).

The performance in terms of the aforementioned parameters is depicted over the swath in Fig. 17. The $2.0 \mathrm{~m}$ resolution goal is achieved over the $400 \mathrm{~km}$ swath with an AASR better than -30.2 $\mathrm{dB}$ and a RASR better than $-26.4 \mathrm{~dB}$. The local AASR fluctuations in Fig. 17 (a) are related to the range-dependent changes in the azimuth sampling due to the different positions of the Tx-induced gaps. The local minima correspond to ranges in which no pulses are lost, whereas the performance is worst for positions in which the gaps are larger.

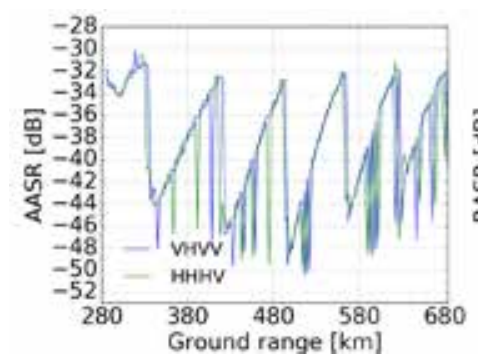

(a)

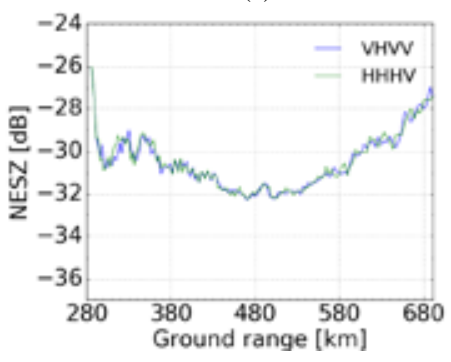

(c)

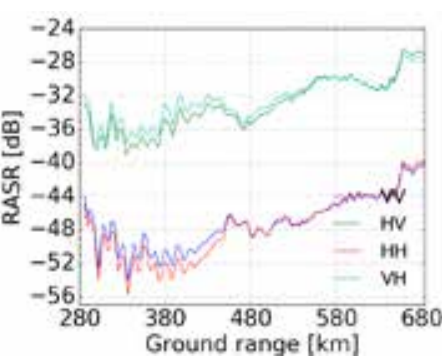

(b)

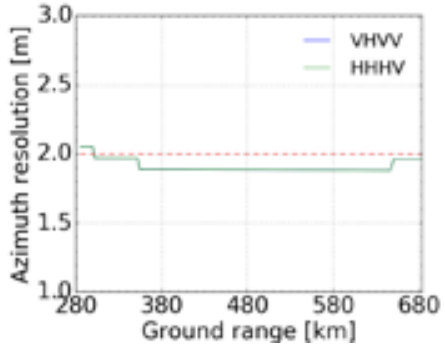

(d)
Fig. 17: Key performance parameters over a $400 \mathrm{~km}$ swath for a quad-pol mode with a reflector SAR system. (a) shows the AASR, (b) the RASR, (c) the NESZ and (d) the azimuth resolution. Different polarizations are highlighted in the picturesin different colors.

The NESZ, better than $-26.0 \mathrm{~dB}$, shows a system with very good sensitivity in spite of the selected azimuth beamforming method (the MSE-VBS solution, not particularly aimed at preserving or enhancing the SNR) and the moderate average transmit power, owing to the large reflector's gain.

\section{REVIEW AND FINAL REMARKS}

This paper introduced novel methods to combine the usage of staggered SAR and multichannel system architectures in azimuth, enabling new imaging modes with added flexibility. The digital beamforming procedures discussed transform the multichannel periodically non-uniformly sampled input signal into an equivalent single-channel signal sampled regularly at a higher rate, using antenna beamforming concepts and a "virtual" array manifold, extended by the combination of information not only from different Rx channels but also from additional pulses. This allows exploiting the channel diversity to increase the 
azimuth resolution while still benefiting from wide gapless range swaths, provided by the staggered PRI operation with multiple simultaneous elevation beams.

It was shown that the new mode and its associated processing enables promising HRWS performance scenarios both for single and multiple polarizations for reflector antenna architectures.

The use of additional channels adds costs and complexity to the resulting systems, and the resulting data rate is identified as a critical point with current technology. As the beamforming uses manifold information, proper calibration of the systems is also an underlying assumption. Further work on the topic thus includes the impact of residual pattern uncertainties and the incorporation of data reduction techniques to the framework. An analysis of the use of the VBS techniques for HRWS systems with a planar phased array antenna will be the subject of a follow-on publication.

\section{REFERENCES}

[1] A. Moreira et al., "Tandem-L: A Highly Innovative Bistatic SAR Mission for Global Observation of Dynamic Processes on the Earth's Surface,” IEEE Geoscience and Remote Sensing Magazine, vol. 3, no. 2, pp.8-23, 2015.

[2] M. Shimada, "JAXA Earth Observation Programs Digest," in IEEE Geoscience and Remote Sensing Magazine, vol. 2, no. 2, pp. 47-52, June 2014.

[3] J. P. Hoffman, S. Shaffer, D. Perkovic-Martin, "NASA L-SAR instrument for the NISAR (NASA-ISRO) Synthetic Aperture Radar mission,” in Proc. SPIE 9881, Earth Observing Missions and Sensors: Development, Implementation, and Characterization IV, 988108, May 2, 2016.

[4] G. Krieger et al., "Advanced Concepts for Ultra-Wide-Swath SAR Imaging," in 7th European Conference on Synthetic Aperture Radar (EUSAR), Friedrichshafen, pp.1-4, 2008.

[5] N. Gebert, G. Krieger and A. Moreira, "Digital Beamforming on Receive: Techniques and Optimization Strategies for High-Resolution Wide-Swath SAR Imaging," IEEE Transactions on Aerospace and Electronic Systems, vol. 45, no. 2, pp.564-592, 2009.

[6] A. Freeman et al., "The "Myth" of the minimum SAR antenna area constraint," IEEE Transactions on Geoscience and Remote Sensing, vol. 38, no. 1, pp. 320-324, Jan 2000.

[7] I. Sikaneta, C. H. Gierull and D. Cerutti-Maori, "Optimum Signal Processing for Multichannel SAR: With Application to HighResolution Wide-Swath Imaging," IEEE Transactions on Geoscience and Remote Sensing, vol. 52, no. 10, pp. 6095-6109, 2014.

[8] S. Huber, A. Patyuchenko, G. Krieger and A. Moreira, "Spaceborne Reflector SAR Systems with Digital Beamforming," IEEE Transactions on Aerospace and Electronic Systems, vol. 48, no. 4, pp.3473-3493, 2012.

[9] G. Krieger et al., "SIMO and MIMO System Architectures and Modes for High-Resolution Ultra-Wide-Swath SAR Imaging," in 11th European Conference on Synthetic Aperture Radar (EUSAR), pp. 1-6., Hamburg, 2016

[10] M. Younis et al., "Techniques and Modes for Multi-Channel SAR Instruments," in 11th European Conference on Synthetic Aperture Radar (EUSAR), pp. 1-6, Hamburg, 2016.
[11] M. Villano, G. Krieger and A. Moreira: "Staggered SAR: HighResolution Wide-Swath Imaging by Continuous PRI Variation," IEEE Transactions on Geoscience and Remote Sensing, vol. 52, no.7, pp.4462-4479, 2014.

[12] G. Krieger et al., "Advanced L-Band SAR System Concepts for HighResolution Ultra-Wide-Swath SAR Imaging" in $5^{\text {th }}$ Workshop on Advanced RF Sensors and Remote Sensing Instruments, (ARSI 2017), Noordwijk, 2017.

[13] M. Suess and W. Wiesbeck, "Side-looking synthetic aperture radar system,” European Patent EP 1 241487, Sept., 2002.

[14] G. Krieger, et al:, "Advanced concepts for high-resolution wide-swath SAR imaging," in 8th European Conference on Synthetic Aperture Radar (EUSAR), Friedrichshafen, Germany, pp. 1-4, 2008.

[15] M. Villano, "Staggered Synthetic Aperture Radar", Ph.D. dissertation, Institute of Radio Frequency Engineering and Electronics (IHE), Karlsruhe Institute of Technology (KIT), Karlsruhe, Germany, February 2016.

[16] M. Villano, G. Krieger and A. Moreira, “A Novel Processing Strategy for Staggered SAR,” IEEE Geoscience and Remote Sensing Letters, vol. 11, no. 11, pp.1891-1895, 2014

[17] F. Queiroz de Almeida and G. Krieger, "Multichannel Staggered SAR Azimuth Sample Regularization," in 11th European Conference on Synthetic Aperture Radar (EUSAR), pp. 1-6, Hamburg, 2016.

[18] F. Queiroz de Almeida, M. Younis, G. Krieger, F. López-Dekker and A. Moreira, "Synthetik-Apertur-Radarverfahren" German Patent 102016208899 , May 2016.

[19] C. Balanis, "Arrays: Linear, Planar, and Circular," in Antenna Theory: Analysis and Design, New York: John Wiley \& Sons Inc., 1997.

[20] S. Bertl, P. Lopez-Dekker, M. Younis and G. Krieger, "Along-track SAR interferometry using a single reflector antenna," IET Radar Sonar \& Navigation, vol. 9, no. 8, pp. 942 - 947, October 2015.

[21] H. L. V. Trees, Optimum Array Processing, New York: John Wiley \& Sons Inc., 2002.

[22] TICRA, “GRASP Software”, Copenhagen, Denmark. Available: http://www.ticra.com/products/software/grasp.

[23] J. Liu et al., "Adaptive beamforming with sidelobe control: a secondorder cone programming approach," in IEEE Signal Processing Letters, vol. 10, no. 11, pp. 331-334, Nov. 2003.

[24] S. Huber et al., "Tandem-L: A Technical Perspective on Future Spaceborne SAR Sensors for Earth Observation" in IEEE Transactions on Geoscience and Remote Sensing, submitted for publication.

[25] M. Villano, G. Krieger and A. Moreira "Ambiguities and image quality in staggered SAR" in 5th Asia-Pacific Conference on Synthetic Aperture Radar (APSAR), Singapore, pp. 204-209, 2015.

[26] D. D'Aria et al., "A wide swath, full polarimetric, L band spaceborne SAR," in IEEE Radar Conference, Rome, pp. 1-4., 2008.

[27] M. Villano, G. Krieger and A. Moreira, "An Analytical Phase-Only Beam Shaping Method for High-Resolution Wide-Swath SAR" in IEEE Geoscience and Remote Sensing Letters, unpublished.

[28] M. Younis, P. López-Dekker and G. Krieger, "Signal and noise considerations in multi-channel SAR" in 16th International Radar Symposium (IRS), Dresden, pp. 434-439., 2015. 


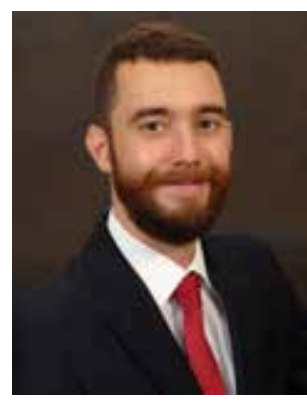

Felipe Queiroz de Almeida received his B.S.E.E. (with honors) and M.S.E.E. degrees in electronic engineering from the Aeronautics Technological Institute (ITA), São José dos Campos, Brazil, in 2009 and 2011, respectively. $\mathrm{He}$ is currently pursuing a Ph.D. in the Karlsruhe Institute of Technology, Germany.

In 2009, he was a research intern in the Microwaves and Radar Institute of the German Aerospace Center (DLR-HR), where he developed his Bachelor Thesis. From 2011 to 2014, he was with Bradar Indústria S.A. in São José dos Campos, working on software development for Synthetic Aperture Radar applications. Since November 2014, he is again with the DLR-HR in Oberpfaffenhofen, Germany, as a Ph.D. Student and research assistant. His research interests include radar signal processing and digital beamforming, in particular azimuth beamforming techniques for High-Resolution Wide-Swath SAR.

Mr. Queiroz de Almeida was awarded the EADS Argus Student Award 2010 for his Bachelor Thesis and the FranzXaver-Erlacher Award for young scientists in 2015.

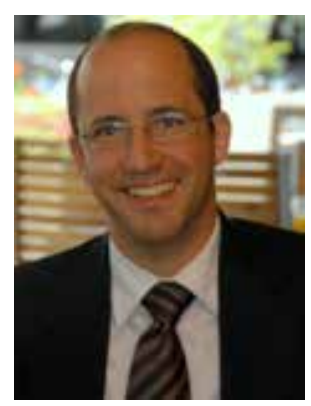

Marwan Younis (S'95, M’05, SM'08) received his B.Sc in electrical engineering from the University of Baghdad, Iraq in 1992 and the Dipl.Ing. (M.Sc.) and Dr.-Ing. (Ph.D.) degree in electrical engineering from the Universität Karlsruhe (TH), Germany, in 1997 and 2004, respectively.

From 1998 to 2004, he was a research scientist with the Institut für Höchstfrequenztechnik und Elektronik, Universität Karlsruhe. Since 2005 he has been with the Microwaves and Radar Institute of the German Aerospace Center (DLR), Oberpfaffenhofen, Germany. He is currently Head of the SAR Techniques Group at the DLR and Professor for Spaceborne Radar Systems at the Karlsruhe Institute for Technology (KIT), Karlsruhe, Germany. He is the author and co-author of about 150 conference papers, and 30 reviewed publications. His research fields include synthetic aperture radar (SAR) systems and techniques, MIMO SAR, digital beamforming, SAR performance, calibration, and antennas. In 1996 he was an intern at the Jet Propulsion Laboratory (JPL) and in 2013 he spent a three month research sabbatical at JPL.

Dr. Younis is an active member of the IEEE and currently director of Corporate Relations for the GRSS. He co-chairs the Instrumentation and Future Technologies GRSS Technical Committee. He is associate editor for the IEEE geoscience and remote sensing letter and reviewer of IEEE publications. He received the Hermann-Billing award for his Ph.D. thesis in 2005.

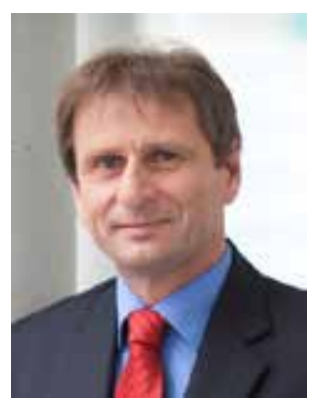

Gerhard Krieger (M’04, SM'09, F'13) received the Dipl.-Ing. (M.S.) and Dr.-Ing. (Ph.D.) degrees in electrical and communication engineering from the Technical University of Munich, Germany, in 1992 and 1999, respectively.

From 1992 to 1999, he was with the Ludwig Maximilians University, Munich, where he conducted multidisciplinary research on neuronal information processing in biological and technical vision systems. Since 1999, he has been with the Microwaves and Radar Institute of the German Aerospace Center (DLR), Oberpfaffenhofen, Germany, where he developed signal processing algorithms for a novel forward-looking imaging radar system employing digital beamforming on receive. From 2001 to 2007 he led the New Synthetic Aperture Radar (SAR) Missions Group which pioneered the development of advanced bistatic and multistatic radar systems, as exemplified by the TanDEM-X mission, as well as innovative multichannel SAR techniques and algorithms for high-resolution wide-swath SAR imaging. Since 2008, he has been Head of the Radar Concepts Department which hosts about 40 scientists working on new SAR techniques, missions and applications. Gerhard Krieger has also been serving as Systems Engineer for the TanDEM-X mission and, since 2013, as a Visiting Lecturer at the Friedrich Alexander University in Erlangen.

Gerhard Krieger is a Fellow of IEEE and received several national and international awards, including two Transactions Prize Paper Awards of the IEEE Geoscience and Remote Sensing Society and the 2012 W.R.G. Baker Award from the IEEE Board of Directors. In 2012, he and his colleagues were nominated for the German President's Federal Award for Innovation and Future Technologies. 


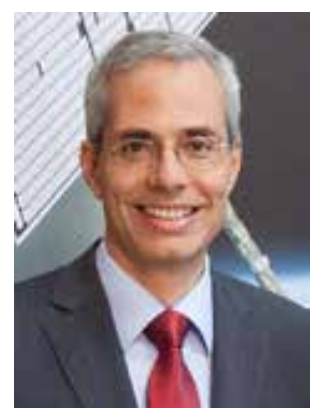

Alberto Moreira (M'92, SM'96, F'04) received the B.S.E.E. and M.S.E.E. degrees from the Aeronautics Technological Institute (ITA), São José dos Campos, in 1984 and 1986, respectively, and the Eng. Dr. degree (with honors) from the Technical University of Munich, Germany, in 1993.

From 1996 to 2001, he was the Chief Scientist and Engineer with the SAR Technology Department, German Aerospace Center (DLR), Oberpfaffenhofen, Germany. Under his leadership, the DLR airborne SAR system has been upgraded to operate in innovative imaging modes like polarimetric SAR interferometry and SAR tomography. Since 2001, Prof. Moreira is the Director of the Microwaves and Radar Institute at DLR and a Full Professor with the Karlsruhe Institute of Technology (KIT), Germany, in the field of microwave remote sensing. His DLR's Institute contributes to several scientific programs and projects for airborne and spaceborne SAR missions like TerraSAR-X, TanDEM-X, SAR-Lupe and SAR-Lupe follow-on as well as Sentinel-1, BIOMASS and Tandem-L. The mission TanDEM-X, led by his Institute, has generated a global, high-resolution digital elevation model of the Earth with unprecedented accuracy. Prof. Moreira is the initiator and Principal Investigator for this mission. His professional interests and research areas encompass spaceborne radar end-to-end system design, analysis and operation, innovative microwave techniques and system concepts, signal processing, and remote sensing applications.

Prof. Moreira is author or co-author of more than 350 publications in international conferences and journals, 8 book chapters and is holder of 20 patents in the radar and antenna field. He is an IEEE fellow and has served as President of the IEEE Geoscience and Remote Sensing Society (GRSS) in 2010. He is recipient of several international awards including the IEEE AESS Nathanson Award (1999) for the "Young Radar Engineer of the Year", the IEEE Kiyo Tomiyasu Field Award (2007), IEEE W.R.G. Baker Award from the IEEE Board of Directors (2012), and the IEEE GRSS Distinguished Achievement Award (2014). He was founder and chair of the GRSS German Chapter (2003-2008), served as Associate Editor for the IEEE GRS Letters (2003-2007) and for the IEEE TGRS (since 2005). He and his colleagues received the GRSS Transactions Prize Paper Awards in 1997, 2001, and 2007 and the GRSS Letters Prize Paper Award in 2015 and 2017. Since 2012 he is serving as the Principal Investigator for the Helmholtz Alliance "Remote Sensing and Earth System Dynamics", comprising of 18 research institutes and 30 associated international partners. 